[Aus dem hygienischen Institut der Universität Breslau.]

\title{
Über die quantitativen Verhältnisse
}

der Tröpfchenausstreuung durch hustende Phthisiker.

Von

Dr. med. H. Ziesché in Breslau,

Assistenten der medizinischen Klinik, früherem Assistenten des Instituts.

Die Möglichkeit einer Übertragung der Tuberkulose von Mensch zu Mensch hat man vorzugsweise gefolgert aus dem Frgebnis von Versuchen an Tieren, die in ähnlicher Weise wie der Mensch für Tuberkulose empfänglich sind. Insbesondere konnten Meerschweinchen, die am meisten zu derartigen Versuchen verwendet worden sind, auf die verschiedenste Weise infiziert werden, durch direkte Einimpfung der Tuberkelbazillen, durch Fütterung und durch Aufnahme der Krankheitserreger in den Inspirationstraktus. Speziell Experimente mit der Inhalation von Sputum oder Kultur haben, von Tappeiner (1) angefangen, eine groBe Anzahl von Forschern in der mannigfaltigsten Weise variiert, so daB kein Zweifel mehr darūber besteht, daB auch durch Einatmung der trocken oder feucht verstäubten Tuberkelbazillen die Erkrankung der Tiere zustande kommen kann. Die am Meerschweinchen gewonnenen Erfahrungen dürfen vermutlich mit der nötigen Vorsicht auf den Menschen übertragen werden. Es ist anzunehmen, daß die Empfänglichkeit für Tuberkulose beim Menschen sicher nicht geringer ist, als beim Meerschweinchen. Während man nämlich nach den bekannten Zusammenstellungen der neuesten Zeit [Franz (2), Naegeli (3)] beim Menschen außerordentlich häufig Tuberkulose findet, ist spontane Tuberkulose beim Meerschweinchen nach dem übereinstimmenden Urteile aller erfahrenen Beobachter auBerordentlich selten, so daB das Überwiegen der Tuberkulose beim Menschen kaum allein durch die gröBere Respirationskapazität und durch die ausgedehntere Infektionsgelegenheit (Husten und Auswurf der Erkrankten) erklärt werden 
kann, sondern daB wohl auch die besonders verbreitete Empfänglichheit eine Rolle dabei spielen muB. - AuBerdem besitzen wir aber eine fast unübersehbare Kasuistik von Übertragungen von Mensch auf Mensch; einzelne dieser Fälle haben fast den Wert von Experimenten. Es erscheint daher kaum mehr notwendig, Beweise für die Übertragbarkeit der tuberkulösen Erkrankungen zu erbringen. Dagegen herrschen noch groBe Meinungsverschiedenheiten über die Art und Weise, in weleher diese Übertragung in der Mehrzahl der Fälle erfolgt.

Eine kurze Übersicht der verschiedenen Möglichkeiten der Infektion zeigt uns, daB teils durch Wunden die Bazillen direkt in die Gewebe gelangen und lokale oder in seltensten Fällen auch allgemeine Tuberkulose verursachen können. Hierher gehören Infektionsversuche aus vorbakterieller Zeit von Villemin (4) and die Beobachtungen vieler anderer späterer Forscher, wie die von Fiselsberg (5), Holst (6), Merklen (7) Tscherning (8) u. a. - Teils erfolgt zweifellos in einer Anzahl von Fällen die Infektion vom Darm aus durch Perlsuchtbazillen in Milch und Butter.

Doch haben diese Infektionsarten anscheinend geringere Bedeutung für die natürliche Verbreitung der wichtigsten tuberkulösen Erkrankung, der Lungenphthise.

Die verbreitetste Infertionsquelle ist oftenbar das Sputum der Phthisiker, und Infektionen mit diesem können zunächst dadurch zustande kommen, daB Kontakte die Übertragung von Sputumteilchen - feucht oder trocken - auf die Schleimhaut des Mundes oder der Nase Gesunder bewirken. Die Kontakte können sich dabei in der Weise vollziehen, daß die Bazillen mit Fingern oder Gebrauchsgegenständen direkt in den Mund gebracht werden, wie die Untersuchungen von Volland (9), Bald win (10), Dieudonné (11) gezeigt haben. Bei Kindern spielt diese Art der Infektion sicher eine nicht zu unterschätzende Rolle, die durch Erziehung zur Reinlichkeit vielleicht erheblich eingeschränkt werden kann. Bei Erwachsenen hat dieser Ansteckungsmodus eine geringe Bedeutung; denn auch bei weniger reinlichen Menschen, bei denen eine Berührung der Nasen- oder Mundschleimhaut mit schmutzigen Fingern öfters vorkommen mag, ist doch die Intensităt des Kontaktes zu gering und die Dauer der Berührung zu kurz, als daB - abgesehen von Ausnahmefällen - eine Ablösung der Keime von infizierten Fingern öfters zustande kommen könnte.

Zweitens können die Tuberkelbazillen mit der eingeatmeten Luft in Mund und Rachen oder nach dem Verschlucken in den Darm gelangen und von da auf Lymphbahnen zur Lunge vorrücken.

Drittens werden eingeatmete Tuberkelbazillen direkt bis in die feineren Bronchien aspiriert. 
$\mathrm{Ob}$ die eingeatmeten Tuberkelbazillen mehr Gefahr auf dem einen oder auf dem anderen Wege bedingen und welcher bäufiger eingeschlagen wird, darüber müssen noch weitere Untersuchungen entscheiden. Hier ist zunächst nur daran festzuhalten, daB die tuberkelbazillenhaltige eingeatmete Luft Infektionen veranlassen kann. Dabei ist aber die Form noch genauer zu berücksichtigen, in welcher sich die Tuberkelbazillen in der Luft vorfinden.

Einmal ist es bazillenhaltiger feinster Staub, der durch leichte Luftbewegungen in die Höhe und zum Schweben gebracht und so eingeatmet werden kann [Cornet (12), Tappeiner(1)]. In die Staubform können die Bazillen übergeführt werden entweder durch Austrocknung und Verstäubung von sorglos auf den FuBboden ausgeworfenem Sputum oder durch feinste Fasern von Taschentüchern und Kleidern, die mit Sputum verunreinigt sind und die dann Gelegenheit gehabt haben, völlig auszutrocknen; oder durch präformierten feinsten Staub, der durch ausgehustete Sputumtröpfchen mit Tuberkelbazillen beladen ist.

Zweitens finden sich die Tuberkelbazillen in der Luft in Form von feinsten Tröpfchen, die rom Phthisiker beim Husten verschleudert werden [Flügge (13), Laschtschenko (18), Heymann (19)].

Es ist hygienisch nicht gleichgũltig, ob die Luft hãufiger und mehr Gefahr bietet durch infektiöse trockene Stäubchen oder Tröpfchen. In dieser Beziehung vertreten Cornet und Flügge mit seinen Schülern den entgegengesetzten Standpunkt. DaB auch trockener Sputumstaub infektionsfähig sein kann, hat Flügge nicht geleugnet, aber nach seiner Ansicht sind die positiv ausgefallenen Experimente unter solchen Bedingungen angestellt, daß sich aus ihnen kein Schluß auf die Häufigkeit dieses Infektionsmodus in praxi ziehen läßt.

Das Sputum war künstlich getrocknet, die in Verwendung gekommenen Sputummengen waren sehr groß; die Loslösung der Stäubchen wurde durch übertrieben kräftige Manipulationen bewirkt und zur Verbreitung der Stäubchen wurden Luftströme angewandt, wie sie in Wohnstuben garnicht vorkommen. Sticher (15) gelang es nur bei Luftströmungen von $1^{\mathrm{m}}$ pro Sekunde, das gesetzte Ziel zu erreichen. Bei Strömungen von 10 bis $30^{\mathrm{cm}}$ pro Sekunde, also Luftbewegungen, die noch lebhafte Zugempfindung hervorrufen, gelang es in keinem Falle mehr, bei den Versuchstieren Inhalationstuberkulose zu erzeugen.

Im täglichen Leben hat nur das am Fußboden angetrocknete Sputum Aussicht durch kräftige Reibung losgelöst und des weiteren verstäubt zu werden. Bei dem gesteigerten Sinne für Reinlichkeit, der sich fortschreitend im öffentlichen Leben wie im Wohnhaus kundgibt und welche sicherlich zum Teil eine Folge der so populär gewordenen Cornetschen 
Prophylaxebestrebungen ist, ist angetrocknetes Sputum nur verhältnismäßig selten an Stellen des Fußbodens zu finden, von wo es durch stärkere Kräfte, wie z. B. beim Gehen losgelöst werden könnte. Häufiger findet es sich an Kleidern und vor allem an Taschentüchern. Doch müssen, wie die experimentellen Untersuchungen gezeigt haben, diese völlig trocken sein, ehe eine Loslösung von Staubteilchen statthat; und in diesen Zustand kommt ein täglich benutztes und in normalen Abständen erneuertes Taschentuch selten.

Die häufige Bildung feinster tuberkelhaltiger Tröpfehen beim Husten und die leichte Infizierbarkeit von Versuchstieren durch solche Tröpfchen ist dagegen von allen bestätigt, die nach Flügge experimentell über diese Frage gearbeitet haben. Auch Cornet (12) hat die Bedeutung der Tröpfeheninfektion nicht etwa auf Grund abweichender Versuchsergebnisse, sondern nur auf Grund einer völlig unrichtigen Berechnung angezweifelt, in welcher er die gesamten in einem Sputum enthaltenen Tuberkelbazillen ohne weiteres als auf feinste flugfähige Stäubchen verteilt in Ansatz bringt, während er selbst früher wiederholt betont hat, wie schwer sich Sputum in wirklich staubförmige Teilchen zerlegen läBt und wie selten dies unter natürlichen Verhältnissen möglich ist. - Die experimentellen Nachprüfungen von Engelmann (21), Fraenkel (22), Moeller (23), Koeniger (24) u. a. haben dagegen die Anschauungen der Flüggeschen Schule durchaus bestätigt. Ihnen gesellen sich aus neuerer Zeit noch einige weitere, weniger bekannte Arbeiten hinzu, auf die ich hier hinweisen möchte. So teilt Petterson (16) mit, daß von 10 Meersehweinchen, welche in einem mit Tuberkelbazillen enthaltenden Sputumstaub versetzten Käfig gehalten wurden, nur 1 tuberkulös wurde; demgegenüber wurden alle 150 Meerschweinchen, welche Petter on Tuberkelbazillen enthaltende zerstäubte Flüssigkeitspartikelchen einatmen lieB, nach einem Monate tuberkulös, und mit einer Nährbodenplatte bekleidete und vor dem Munde Tuberkulöser befestigte Spiegelplatten wurden in 92 Prozent dureh die lediglich beim Husten projizierten Partikelchen (23 unter 25 Fällen) mit Tuberkelbazillen verunreinigt.

In einer früheren Versuchsreihe ließ derselbe Autor (44), um die Flügg e sche Tröpfchentheorie zu studieren, Patienten während des Hustens einen Spiegel, mit drei angehefteten Deckgläschen versehen, eine Fläche von $16^{\mathrm{qcm}}$ bildend, in 10 bis $15^{\mathrm{cm}}$ Entfernung vor den Irund halten. 27 von 29 Versuchen fielen positiv aus. Nach einer Exposition von 1 bis 10 Tagen konnte Petterson bis 1000 Bazillen an der Fläche zählen. Mit den Tröpfehen, die während einer Exposition von 7 bis 14 Tagen an einer Glasscheibe von $17: 10^{\mathrm{cm}}$ GröBe haften geblieben waren, ließen sich Meerschweinchen durch Inhalation tuberkulös machen. Ferner versuchte 
Sorgo (31) zu zeigen, wie viel gefährlicher für die Infektion die Tröpfchenverstreuung der Bazillen ist, als deren Niedersinken und späteres Aufwirbeln mit dem Staube. Ein Meerschweinchen erbielt einige Kubikzentimeter physiologischer Kochsalzlösung eingespritzt, welche eine tuberkulöse Patientin einige Minuten angehustet hatte. Tod nach 8 Wochen an miliarer Abdominaltuberkulose. Ein zweites Tier wurde mit dem Staub der Tasche injiziert, in welchem dieselbe Person Taschentuch und Spuckglas zu tragen pflegte. Tod nach 5 Monaten unter so geringen Veränderungen, da $B$ es sehr fraglich erschien, ob das Meerschweinchen überhaupt allein an einer Tuberkulose gestorben sei. „Es bedarf wohl eigentlich keines Beweises, daB eine Infektion von Mensch zu Mensch unter sonst gleichen Verhältnissen die gefahrbringendere sein muB, als ein Infektionsmodus, der die Möglichkeit einer bereits erfolgten hochgradigen Abschwächung, vielleicht sogar Abtötung durch Austrocknung, Belichtung, Einflu $B$ von ungünstigen Temperaturen bietet."

Auch Bing (36) ist in einigen Untersuchungen über die Verunreinigung der Luft mit Tuberkelbazillen im Krankenzimmer der Phthisiker zu dem Ergebnis gekommen, daB die Infektion durch mit dem Staub aufgewirbelte Bazillen gegenüber der direkten Tröpfcheninfektion eine nur untergeordnete Bedeutung hat und bestätigt die Ergebnisse der Forschungen Flügges und seiner Schüler.

AuBerdem sind in den letzten Jahren eine Reihe von Arbeiten erschienen, die auf Grund statistischer Zusammenstellungen oder praktischer Beobachtungen für die Lehre von der Tröpfeheninfektion eintreten.

Erwähnt sei zunächst eine Statistik, die Mosny (29) 1902 veröffentlicht hat und in der er sich mit Infektionen in der Familie beschäftigt. MIosny findet, daB die Übertragung der Phthise von einem Ehegatten auf den anderen häufiger vorkommt, als die von den Eltern auf Kinder oder unter Geschwistern; es scheint also bei besonders naher Berührung, beim Schlafen in einem Bett, die Infektionsgefahr erhöht zu sein. Ferner weist er auf die zweifellosen Übertragungen hin, die in Hospitälern beobachtet sind (von 102 Krankenwärterinnen des Hotel Dieu zu Paris starben 82 an Tuberkulose). Alle diese Übertragungen, die um so häufiger auftreten, je intimer das Zusammenleben mit dem Phthisiker ist und je länger dieses dauert, führt Mosny vorzugsweise auf die Tröpfcheninfektion zurück. In der von Bernard-Mosny (39) verfaBten Monographie über die Tuberkulose heißt es: „... La transmission directe par les gouttelettes bacillifères, que projecte le phthisique quand il tousse . . . nous donne l'explication de l'extrême fréquence de la contagion familiale et plus particulièrement de la contagion conjugale, les risques de contamination étant directement proportionels à la fréquence, à la durée, à l'intimité des contacts." 
Ferner hat Hillier (33) an der Hand der Gesundheitsberichte der Stadt Manchester von 1902 die Wichtigkeit der Tröpfchenverstreuung für die Tuberkuloseverbreitung studiert. Die notwendigen Erhebungen und Untersuchungen wurden von den beamteten Ärzten - medical officers of health vorgenommen, so daß die Statistik das gröBte Vertrauen verdient.

Die gewöhnlichste Quelle der Ansteckung ist ein schwindsüchtiger Verwandter; 330 Fälle von Tuberkuloseübertragung wurden untersucht. Mit Ausnahme von 23 Fällen wurde die Infektion stets auf Husten und Sprechen eines anderen Phthisikers zurückgeführt. "The views, that infection by Tröpfehen infection is a more potent factor in spreading the disease than the infection which arises from desiccated and pulverized sputa, is supported to a considerable degree by this record of Manchester cases."

Zwei Gründe scheinen Hillier für die Bedeutung der Tröpfchen besonders zu sprechen. 1. „Die Bazillen in den kleinen Tröpfehen haben eine hohe Virulenz, denn sie kommen von ihrem natürlichen Nährboden und sind wahrscheinlich infektionstüchtiger als die im Staube, die lange der Austrocknung und wohl auch dem Lichte ausgesetzt waren. 2. Wenn sich die Tröpfchen zu Boden setzen, so bilden sie allerfeinste Stäubchen, die außerordentlich schnell trocknen und daher sehr schnell wieder aufgenommen werden können."

Die Bedeutung dieser letzterwähnten Tatsache hat neuerdings Kirstein (31) experimentell dargetan.

Endlich hat noch Boeg (35) in den ursprünglichen Verhältnissen der Färöer, in Dörfern, in welche die Tuberkulose erst frisch eingebrochen war, den Infektionswegen nachgeforscht. Die Cornetsche Hypothese von der Wichtigkeit der Staubinfektion konnte er nur selten bestätigt finden. „Dagegen desto zahlreichere Fälle, die Flügges Bypothese bestätigen, nämlich $\mathrm{da} \beta$ das frische und virulente, während des Hustens als feine Tropfen in die Luft verspritzte Expektorat der Phthisiker die gewöhnliche Ansteckungsursache sei. In nicht weniger als 262 von den 342 Fällen von Lungenphthisis hat sich nämlich Ansteckung der Art nachweisen Iassen, also in 77 Prozent der Fälle."

Wenn somit auch für das Vorhandensein einer Infektionsverbreitung durch Tröpfchenverstreuung die erbrachten Beweise als vollauf genügend anerkannt werden müssen, so zeigt ihr Ring doch noch eine kleine Lücke. Bisher hatten sich alle Forscher vorzugsweise nur mit der Art der Tröpfchenbildung [Koeniger (24)] und Tröpfchenverstreuung beschäftigt [Heymann (19)] und sich mit der Feststellung begnügt, daß gelegentlich hustende Phthisiker Tröpfchen in reichlicher Menge verstreuen, in denen 
Tuberkelbazillen vorhanden sind. Mit unseren wachsenden Kenntnissen über den quantitativen Gang der Infektion bei verschiedenen Krankheiten, hat aber auch die Frage nach der $\mathrm{Zahl}$ der in dieser Weise ausgestreuten Erreger das gröbte Interesse gewonnen. Wir wissen jetzt, daB das endgültige Urteil über die Bedeutung des einzelnen Infektionsweges vorzugsweise von den Quantitäts verhältnissen der Infektionsgelegenheiten, in diesem Falle also von den in der Atmungsluft zeitweise vorhandenen Bazillen, abhängig ist. Erst quantitative Feststellungen vermögen uns ein richtiges Bild zu geben von der Gefahr, mit welcher speziell der Hustende und Tröpfchen verstreuende Phthisiker seine Umgebung bedroht.

Daher habe ich in einer größeren Anzahl von Versuchen die Zahl der in bestimmter Zeit und im Bereich eines bestimmten Raumes ausgeworfenen Tröpfehen und Tuberkelbazillen bei hustenden Phthisikern zu verschiedenen Tages- und Nachtzeiten bestimmt.

Die Methodik meiner Tröpfchenversuche war im groBen der auch den früheren Versuchen zugrunde gelegten gleich; nur machte der Zweck der Experimente, quantitativ vergleichbare Werte zu erhalten, einige $A b-$ änderungen notwendig.

Ambulante Phthisiker, bei denen Bazillen im Sputum gefunden waren, wurden angewiesen, während einer halben Stunde vorkommenden Falles und ohne jede Anstrengung auf eine $18 \mathrm{~cm}$ im Quadrat messende Glasplatte zu husten, die vertikal in einer Entfernung von $40-80^{\mathrm{cm}}$ vor ihnen in Mundhöhe aufgestellt bzw. aufgehängt war. ${ }^{\not}$ Darauf wurde diese Glasplatte sorgfältig getrocknet, in Äther-Alkohol fixiert und in toto nach der bekannten Weise gefärbt. Da bei der groBen Ausdehnung der Glasplatte ein Erwärmen des zur Anwendung kommenden Karbolfuchsins nicht möglich war, so wurde sie durch längere Zeit, 6-9 Stunden, in die kalte Farbe gelegt. Darauf erfolgte die Entfärbung und Gegenfärbung mit Korallinmethylenblau. Kontrollversuche hatten die Brauchbarkeit der Methode erwiesen. Nachher wurden die großen Platten in kleinere Stücke zerschnitten und mikroskopisch untersucht.

Die Unbequemlichkeiten, die das Arbeiten mit solehen großen Platten im Gefolge hatte, führten bald zu folgender Verbesserung der Methode: An Stelle der großen Platten traten zwölf Objektträger englischen For-

1 Das Auffangen der Tröpfchen mittels vertikaler Platten ist unvollständig; nachweislich werden feinste Tröpfchen von diesen vielfach zurückgeschleudert und nicht fixiert. Das Auslegen horizontaler Platten war aber für meine Versuche noch weniger brauchbar, weil alsdann gleichmäßige Bedingungen za schwer herzustellen sind. Schließlich mußte ich den gleichmäBigeren vertikalen Platten den Vorzug geben, bei diesen aber vorkommendenfalls den nicht fixierten Anteil der Tröpfehen in Rechnung ziehen. 
mates, die, in einen passenden Blechrahmen gespannt, genau die gleiche Größe von $324 \mathrm{gm}$; hatten. Auch die Färbung konnte nunmehr nach der alten Zielschen Methode: Erhitzen in konzentriertem Karbolfuchsin, Entfärben in 5proz. Schwefelsäure und Nachfärben mit Methylenblau 1:5000 vorgenommen werden. Die Nachfärbung mit Korallinmethylenblau hatte sich weniger bewährt, weil sich leicht wolkige Niederschläge bildeten, welche die Übersicht außerordentlich erschwerten. Nach dieser Behandlung erscheinen die Objektträger auf weißer Unterlage rein durchsichtig, während sich die Tröpfchen, je nach ihrer Herkunft aus dem Munde oder tieferen Teilen als schwach blaue, unbestimmt umgrenzte oder tief blaue Kreise mit scharfer Grenze darstellten.

Heymann (19) hat in seiner ersten Arbeit eine Schilderung der Morphologie der Tröpfchen gegeben, der nur noch wenig hinzuzufügen ist.

Er unterscheidet drei Typen von Tröpfchen; ein Normaltypus besteht aus drei Schichten, einem zentralen Kern aus Fibrin und Leukozyten, einem konzentrischen Kreise aus Mundepithelien gebildet und einem zweiten aus schwach gefärbtem Schleime mit wenig stärker gefärbten Fäden sich zusammensetzend. Davon abgeleitet findet sich eine zweite Art, die fast nur aus dem leukozytenreichen Kern, mit schmaler Schleimschicht besteht, und eine dritte, die nur aus Epithelien und Schleim sich zusammensetzt. Wenn man nur eine Einteilung auf Grund der Formelemente vornehmen und dabei vor Ausnahmen nicht zurückschrecken will, so ist diese Einteilung wohl genügend. Zieht man es indes vor, genetisch die Entstehung der Tröpfchen zu verfolgen, so wird man eine andere Einteilung anwenden.

Die für uns wichtigen Tropfen sind die zumeist bazillenhaltigen, welche dem Heymannschen Typus II entsprechen. Sie zeigen sich als kleine bis mittelgroße Tröpfchen von bald runder, bald ovaler Form. Sie enthalten keine Mundepithelien und fast nie Begleitbakterien, wie Streptokokken und Diplokokken, aber fast stets Tuberkelbazillen in größerer oder kleinerer Anzahl. Häufig sind überraschend viele Bazillen in einem Tröpfchen zu zählen; in einem Falle fand ich Tröpfchen, die bei starker VergröBerung - Zeiss 0c. 4, Olimmersion - 6, 14 und 17 Gesichtsfelder groß waren und 433, 373 und 587 Bazillen enthielten. Vermischen sich dieseTröpfchen, die direkt aus den Bronchien stammen und die ich daher im folgenden als Bronchialtröpfchen bezeichnen werde, mit Speichel, und schlagen sie senkrecht auf die Unterlage auf, so entsteht der Heymannsche Normaltypus, den ich auch, aber nur relativ selten, zu beobachten Gelegenheit hatte. Durch innigere Vermischung der Bronchialtröpfehen mit Speichel im Munde entsteht Heymanns Typus III. Diese 
letzten beiden Gruppen stelle ich als Mundtröpfchen den reinen Bronchialtröpfchen gegenüber.

Von der gröBten Wichtigkeit sind gerade diese Bronchialtröpfchen, die im Achsenstrome des Bronchus fortgerissen mit groBer Geschwindigkeit und Kraft ohne jeglichen Aufenthalt im Munde in die Außenluft gelangen. Sie haben eine gröBere Propulsivkraft als die Mundtröpfchen, wie in einer Röhre mit strömender Flüssigkeit der Axialfaden eine gröBere Geschwindigkeit hat als die Randzonen.

Man kann dies leicht demonstrieren; hat man einen gut verstreuenden Patienten, der indes infolge groBen Speichelreichtums auch viele Mundtrōpfchen verbreitet, so braucht man nur die Entfernung der Vorlage vom Hustenden za vergrößern. Die trägeren Mundtropfen, die infolge ihrer GröBe auch schwerer sind, bleiben dann zurück und fallen zu Boden, während die Bronchialtröpfchen das Glas erreichen und nunmehr vorherrschen.

Ein anderer Beweis für die Richtigkeit dieser Auffassung liegt in der Verteilung der Tröpfchen dem Raume nach. Auf der vorgestellten Glasplatte findet man die Bronchialtröpfchen ziemlich regelmäBig im Zentrum des Verstreuungskreises, während die Mundtröpfchen sich an der Peripherie anhäufen. Natürlich wird durch Veränderung der Kopfhaltung und der Mundstellung dieses Verhalten oft verwischt.

Die Mundtröpfchen sind zum kleinsten Teile bazillenhaltig. Die bazillenhaltigen Tröpfchen entstehen, wenn Bronchialtröpfchen auf ihrem Wege durch den Mund sich mit Speichel vermischen und rasch beim nächsten Hustenstoße wieder herausgeschleudert werden. Dis meisten Mundtröpfchen sind aber bazillenfrei und unschuldig; sie enthalten häufig andere Bakterien wie Streptokokken, Diplokokken, Mundstäbchen usw.

Es ist nicht unwahrseheinlich, daß bei der Propagation anderer Infektionskrankheiten wie der Streptokokken-Angina, der Diphtherie usw., bei denen man die Erreger zum Teil beständig und in großer Menge im Munde findet, Mundtröpfchen die Hauptrolle spielen. Bei der Phthise sind sie von untergeordneter Bedeutung.

$\mathrm{DaB}$ auch im Speichel Tuberkulöser Tuberkelbazillen gelegentlich gefunden werden, hat schon Laschtschenko (18) nachgewiesen, und auch ich habe mich daron überzeugt, konnte sie aber viel seltener finden.

Auch Moeller (23) vermochte bei Patienten, die nicht kurz zuvor gehustet hatten, nur selten im reinen Mundspeichel Tuberkelbazillen nachzuweisen. Die geringe Infektiosität der Mundtröpfchen beruht auBerdem darauf, daß die beim Husten verschleuderten Speicheltröpfchen relativ groß sind; sie fallen deshalb und wegen der nur geringen Propulsivkraft, die ihnen innewohnt, bald zu Boden. Die Tuberkelbazillen des Speichels 
stammen von den beim Auswerfen im Munde zurückbleibenden Sputumteilen. Zum gröBten Teile werden diese wieder verschluckt, oder beim nachträglichen Hüsteln und Räuspern noch herausgebracht. Die Auswaschung der Bazillen aus dem meist zähen Sputum geht anscheinend nur langsam und unvollkommen von statten.

Endlich findet man noch eine Tröpfchenform, die Heymann nicht erwähnt hat, nämlich die Hantelform. Diese hat ihre theoretische Wichtigkeit für die Frage, ob die verstreuten Tröpfchen homogener Natur sind oder nicht. Wären sie durchaus homogen, so müBten sie im schiefen Aufprall ellipsoide Formen annehmen, wie das nach Heymanns Erfahrung auch hin und wieder vorkommt. Häufiger kommt es indes zur Bildung der Hantelformen, einem Oval, das durch einen mehr oder minder ausgezogenen Stiel mit einem zweiten Oval in Verbindung steht. Die Entstehung dieser Bildungen ist vermutlich dadurch zu erklären, daB viele Tröpfchen durch die Einschlüsse, die sie enthalten, inhomogen werden und daB nun beim Aufschlagen der schwerere Teil die Tendenz hat, sich weiter fortzubewegen, als der leichtere. Besonders geeignet zur Hantelformbildung sind die kleinen Bläschen mit inhomogener Wandung, die ja auch des öfteren zur Beobachtung kommen und schon von Heymann beschrieben worden sind.

Die Inundtropfen, wie sie durch Sprechen entstehen und deren Genese Ko eniger (24) so ausgezeichnet dargestellt hat, sind also relativ ungefährlich. Damit fällt der Einwand fort, der hin und wieder, besonders von Anstaltsärzten gemacht wurde, daB die Konsequenzen der Tröpfcheninfektion unmenschlich und somit vom medizinisehen Standpunkt zu beanstanden seien.

Die quantitativen Resultate meiner Untersuchung sind in der am Schluß befindlichen Tabelle 1 zusammengestellt. Ich habe im ganzen 30 Patienten (in insgesamt 62 Versuchen) untersucht, von denen 22 mit irgendwelchem bemerkenswerten Befund in der Tabelle registriert sind. Die groBe Mühe und der erhebliche Zeitaufwand, welchen die genaue Durchmusterung derartiger Präparate verlangt, machte es mir leider unmöglich, eine noch größere Anzahl von Kranken zu untersuchen. Versucht man solche Präparate länger als 3 Stunden hintereinander genau zu durchmustern, so übersieht man sicher eine große Anzahl von Bazillen. Überhaupt sind alle gefundenen Bazillenzahlen Minimalwerte. Es geht dies daraus hervor, daB bei wiederholt vorgenommenen Kontrollzählungen, schon früher gezählte Objektträger durch mich oder andere stets mehr Bazillen gefunden wurden. An manchen Stellen liegt auch der Leukozytenteppich so dicht, daB eine groBe Anzahl von Tuberkelbazillen unbemerkt und ungezählt bleibt. Ich glaube, daß man die registrierten Werte noch beträcht- 
lich erhöhen müßte, um den wahren Gehalt an Tuberkelbazillen zu bekommen.

Vielleicht hat auch die allmählich bessere Übung im Untersuchen das bei den wiederholt untersuchten Personen häufig zutage getretene Resultat beeinflußt, daß im zweiten Falle die Bazillen in einem höheren Prozentsatze und in gröBerer Menge gefunden wurden, als bei der ersten Untersuchung.

Das von mir untersuchte Krankenmaterial entstammte in der Mehrzahl der Phthisikerabteilung des hiesigen städtischen Wenzel-Hanckeschen Krankenhauses, dessen Leiter, Herrn Primärarzt Dr. Drewitz, ich für sein liebenswürdiges Entgegenkommen zu größtem Danke verpflichtet bin. Auch Herr Sekundärarzt Dr. Schellschmidt erleichterte mir in jeder Weise mein Vorgehen, wofür ich ihm bestens danke.

Es wurden nur Patienten untersucht, die Tuberkelbazillen im Sputum hatten, doch wurde teine Rücksicht darauf genommen, ob dieselben zahlreich oder spärlich waren. Die Untersuchungen erstreckten sich über einen großen Teil des Jahres und fanden zu jeder Tageszeit statt, da ich mir auch über zeitliche Unterschiede in der Verstreuung der Bazillen ein Urteil bilden wollte.

Von den 30 Personen, die zur Untersuchung kamen, verstreuten $12=40 \cdot 0$ Prozent Bazillen. . Diese Zahl stimmt zufällig überruschend mit der ron Heymann (20) 1899 gefundenen Quote überein, von dessen Versuchspersonen 40.0 Prozent Bazillen ausstreuten.

Interessant ist der Vergleich der einmal mit den öfters untersuchten Personen. Während bei den ersteren in 12.5 Prozent der Fälle Tuberkelbazillen durch Tröpfchen verbreitet wurden, betrug der Prozentsatz bei ofter untersuchten Personen 78.9 Prozent; es verstreuten also fast $4 / 5$ aller mehrmals untersuchten Personen bazillenhaltige Tröpfchen.

Erwähnen möchte ich noch, daB ich in der ersten Zeit meiner Untersuchungen weit schlechtere Resultate hatte als später, und daB ich bei wiederholter Durchmusterung älterer Präparate auch da noch, zum Teil reichlich, Bazillen fand, wo ich zunächst einen negativen Befund notiert hatte. Es mahnt dies zur Vorsicht bei der Anstellung ähnlicher Untersuchungen. Es würde entschieden unrichtig sein, wollte man aus einer Anzahl nur je einmal untersuchter Personen und ohne vorhergehende längere Einübung speziell auf solche Untersuchungen Schlüsse bindender Art über die Frequenz des Tröpfchenverstreuens ziehen. Auf Grund meiner längere Zeit festgesetzten Erfahrungen zweifle ich nicht daran, daB, wie es schon Heymann vermutet hat, jeder Phthisiker in irgend einem Stadium seiner Krankheit einmal Bazillen in flugfähigen Tröpfchen entleert und auf diese Weise Infektionen veranlassen kann. 
Die Untersuchungen von Blume (27) machen es sogar wahrscheinlich, $\mathrm{daB}$ eine gewisse Tuberkelbazillenverstreuung noch erheblich offter bei Phthisikern vorkommt, als man bisher annahm. Blume kọnte nämlich in solchen Fällen, wo ihm wegen mangelnden Auswurfes die bakteriologische Sicherung der Diagnose nicht möglich war, Bazillen noch dadurch nachweisen, daB er die Patienten anhielt, einen Objektträger während 8 bis 10 Tagen jeden Morgen anzuhüsteln. Er fand dann in den fixierten und nach Ziehl gefärbten Präparaten Tuberkelbazillen, bei einigen vereinzelt, bei anderen auch in Konglomeraten in Bronchialtröpfchen. Es scheint danach eine Tröpfchenverstreuung selbst bei den im Anfangsstadium sich befindenden Kranken stattzufinden, deren häufigem Räuspern und Hüsteln ohne kräftigere Hustenstöße man bisher kaum Beachtung geschenkt hat.

Betrachten wir das Verhältnis der Mund - zu den Bronchialtröpfchen, so sehen wir, daB in der Mehrzahl der Fälle die ersteren im Übergewichte sind. In den 62 Versuchen war nur sechsmal das Verhalten ein umgekehrtes (Nr. 13, 49, 50, 51, 59 und 60). Dreimal handelte es sich dabei um akute Katarrhe mit vermehrter Sekretion, flüssigem Sekrete und dadurch augeregtem Hustenreize; dreimal war dies bei einer Patientin der Fall, die bei ausgedehnter Zerstörung des Oberlappens mit weiter Bronchialkommunikation außerordentlich geringe subjektive Symptome zeigte. Bei ihr hatte sich das normale Verhalten umgekehrt. Die Bronchialtröpfchen waren stets in der Mehrzahl.

Die Gesamtanzahl der verbreiteten Tröpfchen ist sehr verschieden und hängt von verschiedenen Faktoren, von der Flüssigkeit oder Zähigkeit des Sekretes, der Kraft des Hustens usf. ab. Im allgemeinen verstreuen ambulante Patienten, die noch wohlauf sind und infolge der, durch die Bewegung erhöhten Zirkulation auch mehr sezernieren, mehr Tröpfchen als schwache, bettlägerige Kranke. Stark fiebernde Phthisiker mit trockenem Hals und Mund verstreuen beinahe gar nicht, aber dann fast nur Bronchialtropfen.

Die Anzahl der verstreuten Bazillen schwankt ebenfalls innerhalb weiter Grenzen. Es sind hier die verschiedenen Umstände, Stadium der Krankheit, Körperkraft, vorangegangene Bewegung, Fieber usf. wïksam, die bereits Heymann in ihrer Bedeutung gewürdigt hat.

Nächst den wenigen Patienten, die auch bei wiederholter Untersuchung keine Bazillen zeigen, zeigen die geringsten Befunde jene, die nur hin und wieder wenig oder mehr Bazillen entleeren, wenn besondere günstige Verhältnisse, wie vermehrte Bewegung, akute katarrhalische Affektionen u. a.m. vorliegen. 
Die kleinste Anzahl der nachgewiesenen Bazillen betrug 3 (20), um in anderen Fällen auf 67 (4), $136(6), 352(25), 952(60), 1445$ (49), ja bis zu der ganz exorbitanten Höhe von 20174 Bazillen in einem Bereich von $324 \mathrm{qem}$ zu steigen.

Die Frau, welche die letzteren Werte (Nr. 49-51) lieferte, zeigte eine weit fortgeschrittene Tuberkulose; fast die ganze obere Hälfte der linken Lunge war zerstört; man hörte in diesem Bereiche feuchtes, groBblasiges Rasseln. Die Frau, früher Zigarrenarbeiterin, war seit 2 Jahren verheiratet, Mutter eines zweijährigen Knaben. Die subjektiven Störungen waren auffallend gering. Die Frau versorgte ihre Häuslichkeit und leistete noch Nebenarbeit als Zigarrendreherin. Sie klagte nun hin und wieder über Schmerzen und Müdigkeit. Nachtschweiße waren wenig vorhanden und wurden nicht störend empfunden. -

Was die Schwankungen im Umfang der Tröpfchenausstreuung und speziell ihre Verteilung nach Tageszeit und Jahreszeit anlangt, so haben wir bisher darüber keine sicheren Angaben. Leider ist auch mein Material nicht groß genug, um die aus ihm gezogenen Schlüsse verallgemeinern zu können. Diese und ähnliche, vielleicht auch praktisch nicht unwichtige Fragen werden wohl erst beantwortet werden können, wenn das große Laterial der Volksheilstätten zu planmäBigen Versuchen verwandt werden wird.

Ich habe meine Untersuchungen in Zwischenräumen von je vier Stunden geordnet und so folgendes Ergebnis bekommen.

Es hatten ein positives Ergebnis bei Versuchen von:

\begin{tabular}{c|c|c}
\hline Zeit & Prozent & Anzahl der untersuchten Fälle \\
\hline \hline $7-11$ Uhr früh & $31 \cdot 2$ & 32 \\
$11-3 "$ & $62 \cdot 5$ & 7 \\
$3-7 "$ & $64 \cdot 7$ & 15 \\
$7-11$, abends & - & 3 \\
$11-3$, & - & 3 \\
$3-7$, & - & 2
\end{tabular}

Wir sehen also, daß von früh um $7 \mathrm{Uhr}$ an die Tröpfchenverstreuung zuerst schnell, dann langsam bis um $7 \mathrm{Ohr}$ abends zunimmt. In späteren Abendstunden und während der Nacht habe ich nur wenige Untersuchungen anstellen können, so daB eine Prozentberechnung nicht zulässig ist. $\mathrm{DaB}$ Nachts und am frühen Morgen wenig Verstreuung beobachtet wurde, das liegt offenbar daran, daB auf der mir zur Verfügung stehenden Krankenabteilung kurz vor dem Schlafengehen den Kranken Narkotika gegeben wurden, um die Nachtruhe zu sichern. Wie prompt die Wirkung 
ist, zeigt der Ausfall unserer Versuche. Gegen früh läBt dann die Wirkung nach. Erst am späteren Morgen macht sich die Reaktion auf die lange Sekretstauung bemerkbar, und mit dem Husten setzt auch stärkere Verstreuung ein.

Bei einem Patienten Hag. (Nr. 27 bis 42), der deutlich, aber in mäBigen Grenzen Tröpfchen verstreute, habe ich wiederholt den EinfluB der Tageszeit im Laufe des Tages auf die Anzahl der ausgehusteten Bazillen festzustellen gesucht. Ein irgendwie gesetzmäBiges Verhalten konnte ich aber dabei nicht finden. Das einzige, was sich mit Sicherheit auch hier bemerkbar machte, war die deutliche Wirksamkeit der abendlichen Morphiumgabe.

Im übrigen wächst offenbar die Tröpfchenverbreitung mit dem Husten, und dieser nimmt nach dem Aufstehen, nach heftiger Bewegung, angeregter Unterhaltung, kurz nach allen Einflüssen zu, die eine Steigerung der Blatbewegung und damit eine Erhöhung der Sekretionsgeschwindigkeit im Gefolge haben. Je nachdem der Zeitpunkt der Untersuchung nahe oder entfernt von einer solchen erhöhenden Ursache liegt, finden sich auch mehr oder weniger bazillenhaltige Tröpfchen auf der Vorlage.

Auch über die Bedeutung der verschiedenen Jahreszeiten und der Witterungsverhältnisse auf die Intensität der Tröpfchenverstreuung konnte ich wenig Sicheres feststellen. Am Ende des Winters und zu Beginn des Frühjahrs scheinen wohl mehr bazillenhaltige Tröpfchen ausgehustet zu werden; und die Ursache dafür sind wohl die in jenen Zeiten häufig akquirierten Katarrhe der Phthisiker, wie ja auch die Mortalität der Phthisiker in diesen Zeiten aus dem gleichen Grunde erhöht zu sein pflegt.

Unter meinen Patienten war das Verhältnis der Tröpfchenverstreuer zu denen, die keine Bazillen auswarfen, in den verschiedenen Monaten folgendes:

\begin{tabular}{|c|c|c|c|c|}
\hline Monat & & $\begin{array}{l}\text { Insgesamt } \\
\text { untersucht }\end{array}$ & $\begin{array}{c}\text { Bazillenfreie Tröpfchen } \\
\text { verstreuten }\end{array}$ & $\begin{array}{c}\text { Bazillenhaltige Tröpfchen } \\
\text { verstreuten }\end{array}$ \\
\hline Januar & . & 18 & 11 & 7 \\
\hline Februar. & . & 15 & 6 & $\mathbf{9}$ \\
\hline März . . & . & 14 & 5 & 9 \\
\hline April . & . & - & - & - \\
\hline Mai . & . & 1 & 1 & 0 \\
\hline Juni . & . & 3 & 1 & 2 \\
\hline Jnli . & . & 2 & 0 & 2 \\
\hline August . & . & - & - & - \\
\hline September & . & - & - & - \\
\hline Oktober . & . & - & - & - \\
\hline November & . & 8 & 6 & 2 \\
\hline Dezember & . & 2 & 2 & 0 \\
\hline
\end{tabular}


Versucht man nunmehr, die quantitativen Ergebnisse meiner Beobachtungen für die Abschätzung der Infektionsgefahr zu verwerten, welche aus der Tröpfchenverstreuung hustender Phthisiker sich ergibt, so wird es vor allem nötig sein, einen bestimmten Begriff von derjenigen Bazillenzahl zu bekommen, welche zu einer wirksamen Infektion durch Inhalation erforderlich ist. Da diese Zahl sich nur experimentell und daher nicht für den Menschen festlegen läßt, müssen wir uns mit Ermittelungen an Versuchstieren begnügen. Von der Anzahl Bazillen, welche bei diesen zur sicheren Infektion durch Inhalation ausreicht, werden wir unter gewissem Vorbehalt einen RückschluB ziehen können auf die für den Menschen mindestens nötige Ziffer von eingeatmeten Tuberkelbazillen; und es wird sich dann fragen, inwieweit die von mir in feinsten ausgestreuten Tröpfchen hustender Phthisiker gefundene Menge von Tuberkelbazillen zur Deckung dieser Ziffer ausreicht.

Uber das Quantum der zur Infektion von Meerschweinchen erforderlichen inhalierten Bazillen liegen Versuche aus früherer Zeit vor von Gebhard (26) und ron Preyss (27). Beide arbeiteten mit phthisischem Sputum; die Feststellung der Bazillenzahl erfolgte durch mikroskopische Zählung einer genau gemessenen Probe; von den einzelnen Verdünnungen inhalierten die Tiere ein bestimmtes Quantum (Gebhard) oder mittels des gleichen Sprays eine bestimmte Zeitlang (Preyss). Gebhard fand, daB inhalierte Sputumverdünnungen in dieser Weise noch tödliche Tuherkulose rerursachten, wenn etwa 400 Tuberkelbazillen eingeatmet wurden; Preyss fand einmal sogar 36 Bazillen noch wirksam, ein anderes Mal 48 Bazillen unwirksam.

Neuerdings hat Findel mit genaueren Methoden die bei der Inhalation erforderliche Infektionsdosis bestimmt (siehe die Arbeit im vorlieg. Band).

Er hat dabei auch unterschieden diejenige Bazillenmenge, welche in der Atemluft eingeatmet werden muB, und diejenige, welche nach Abzug der in Nase, Rachen usw. zurückbleibenden Bazillen wirklich bis zu den Bronchien vordringen müssen. Der letztere Anteil beträgt etwa 60; zwar gelingt die Infektion auch schon mit kleineren Hengen, aber sie ist unsicher; während 60 eindringende Bazillen in jedem Falle tödliche Tuberkulose veranlassen. Damit 60 Bazillen in die Bronchien gelangen, müssen aber mindestens 200 Bazillen eingeatmet werden, von denen mehr als $2 / 3$ in Nase, Rachen usw. steeken bleiben bzw. rerschluckt werden.

Es fragt sich nun zunächst, ob die für das Meerschweinchen als erforderlich erkannte Dosis auf den Menschen übertragen werden darf.

Hierfür kommt einmal in Betracht, ob der Prozentsatz der aus der Einatmungsluft in Nase, Rachen usw. zurückgehaltenen Keime beim 
Menschen größer ist als beim Meerschweinchen. Bedenkt man den kräftigeren Inspirationsstrom des Menschen, andererseits die außerordentliche Enge der Zugangswege zum Respirationstraktus beim Meerschweinchen, so wird man kaum annehmen können, daß der Mensch in dieser Beziehung besser geschützt sei. - Vielleicht ist aber die Minimaldosis Bazillen, welche nach dem Eindringen in die feineren Bronchien tuberkulöse Erkrankung veranlaßt, beim Menschen, schon wegen des großen Unterschiedes in GröBe und Gewicht des Körpers und der Organe viel erheblicher als beim Meerschweinchen? Auch in dieser Beziehung wird man keine allzu großen Differenzen erwarten dürfen. Für das Schicksal der bis in die feineren Bronchien vorgedrungenen Bazillen hauptsächlich entscheidend ist die sog. Disposition, d. h. die Fähigkeit des Körpers, durch Schutzvorrichtungen der eindringenden Krankheitserreger Herr zu werden. Die Disposition ist aber, wie wir aus der enormen Verbreitung und dem häufigen tödlichen Ende der menschlichen Tuberkulose sehen, beim Menschen außerordentlich groß, vermutlich eher größer als beim Meerschweinchen. Die für letztere als erforderlich ermittelte Einatmungsdosis wird daher für den. Menschen wohl etwas höher liegen, aber gewiß zu einer wirksamen Infeiztion ausreichen, wenn man sie 2 bis 3 mal höher ansetzt. - Andererseits werden minimalste Mengen inhalierter Bazillen auch beim Menschen sicher nicht Infektion bewirken, weil die Chancen, daß sie in die feineren Bronchien gelangen, auf Epithellücken treffen oder in Lymphbahnen eindringen können, zu gering sind.

Rechnen wir demnach, daß auch für den Menschen etwa 2 bis 400 Tuberkelbazillen mit der Atemluft aufgenommen werden müssen, um Infektion zu bewirken; und sehen wir zu, ob die Verhältnisse bei der Tröpfchenverstreuung der Phthisiker nach meinen Versuchen so liegen, daB die Aufnahme von so viel Bazillen seitens eines Gesunden innerhalb einer kurzen Zeitspanne erfolgen kann.

Auf den von mir benutzten Glasplatten von 324 qom fanden sich binnen einer halben Stunde bei 9 Untersuchungen mehr, zum Teil erheblich mehr als 200 Tuberkelbazillen. Durch den menschlichen Inspirationsstrom werden außerdem jedenfalls die verstreuten Tröpfchen aus einem erheblich größeren Luftbereich in die Lunge eingeführt; die Glastafel schneidet aus dem ganzen Verstreuungskreise nur ein relativ kleines Segment heraus.

Andererseits habe ich bei 23 Einzeluntersuchungen in einer halbenStunde keinen Bacillus gefunden; und in 20 Untersuchungen weniger als 200.

Es kommt hinzu, daß die Annäherung meiner Versuchsplatte an den hustenden Phthisiker eine ziemlich groBe war, im Mittel $60^{\mathrm{cm}}$, also größer als gewöhnlich unter praktischen Verhältnissen; ferner daß die 
Platte eine halbe Stunde dauernd dem Husten in der gleichen Entfernung exponiert war, was auch in der Praxis nicht gerade häufig vorkommt.

Weiter ist in Rechnung zu ziehen, daB sicher nur die kleinen Bronchialtröpfchen von ca. 20 bis $60 \mu$ Durchmesser Aussicht haben, bis in die feineren Bronchien zu langen. Eine genauere Scheidung nach der GröBe der Bronchialtröpfchen habe ich in meinen Versuchen unterlassen. Das Verhältnis der feineren zu den gröberen wechselt sehr stark; bei gröBerer Entfernung der auffangenden Platte treten die gröberen mehr zurück. Wie ich oben hervorhob, ist es andererseits sehr fraglich, ob bei etwas gröBerer Entfernung die feinsten Tröpfchen auch wirklich auf den Objektträgern aufgefangen werden; Versuche mit Tröpfchen eines sehr feinen Sprays lassen Zweifel hieran als durchaus berechtigt erscheinen. So sind noch viele Unsicherheiten vorhanden, die eine genauere quantitative Abschätzung der in den Tröpfchen gebotenen Infektionsquelle vorläufig sehr erschweren.

Dennoch wird man sich im allgemeinen über die Gefahr, die von der Tröpfchenverstreuung ausgeht, etwa folgendes Bild machen können.

Sicher kommen außerordentlich selten so reichliche oder so dicht mit Bazillen besetzte kleine Bronchialtröpfchen ror, daB schon ein kurzer Aufenthalt in der Nähe des Phthisikers ausreicht, um die zur Infektion erforderliche Bazillenmenge in die Einatmungsluft zu liefern. Kurzdauerndes Zusammensein mit einem Phthisiker und gelegentliches Anhusten führt daher wohl fast niemals zur Infeltion.

Dagegen wird bei dauerndem nahem Zusammensein die infektiöse Grenzzahl leicht erreicht. Ein halbstündiges Verweilen in nächster Nähe des Phthisikers liefert noch relativ geringe Chancen; in meinen Versuchen 9 mal unter 52. Erst bei noch stärkerer zeitlicher Ausdehnung des intimen Verkehrs werden die Chancen erheblich.

Demnach wird z. B. der Verkehr einer phthisischen Mutter mit ihrem in den ersten Lebensjahren befindlichen Kinde fast unfehlbar zur Infektion des Kindes führen. Es ist unausbleiblich, daß die Mutter täglich stundenlang sich in nächster Nähe des Kindes befindet, und wenn sie auch bei kräftigeren Hustenstößen sich abwendet, so wird diese Maßregel doch so oft von unvollkommener Wirkung sein, und schon das kaum merkliche Hüsteln führt so leicht zu Tröpfehenverstreuung, daß das Kind zweifellos häufig Tröpfchen einatmet. Wohl mag die Mutter monatelang keine Tuberkelbazillen verstreuen; es wird dennoch hier und da zweifellos za einer Periode stärkerer Infektionsgefahr kommen. - Weniger leicht werden schon Ehegatten und andere Familienmitglieder sich infizieren. Der dauernde Aufenhalt in nächster Nähe während des Hustens ist hier 
viel leichter $\mathrm{zu}$ vermeiden und wird entschieden häufiger vermieden. Ebenso ist die gemeinsame Arbeitsstātte nicht durchweg gefährlich, und die Gefahr kann durch entsprechendes Verhalten des hustenden Phthisikers einerseits, des Gesunden andererseits sehr erheblich herabgedrückt werden. - Nicht leicht vermeidbar wird die Tröpfcheninfektion bei dem Pflegepersonal auf Phthisikerstationen sein. Bei der Pflege eines einzelnen Kranken ist der andauernde Aufenhalt in gröBter Nähe noch zu umgehen, etwa ebenso wie bei Ehegatten (ein verständiges Verhalten des Kranken vorausgesetzt!). In Pflegeanstalten mit ambulanten, meist noch kräftigen Kranken braucht ebenfalls eine Infektion des Personals durchaus nicht zustande zu kommen. Dagegen ist bei be ttläg erig en Kranken im letzten Stadium, die fortgesetzt Hilfeleistungen und zu diesen große Annäherung des Pflegepersonals erfordern, die Gefāhrdung des letzteren beträchtlich; und wenn der Pfleger eine große Anzahl solcher hilfloser Kranker zu warten hat, so befindet er sich fast dauernd in einer stark mit infizierenden Tröpfchen erfüllten Luft. - Ärzte sind relativ wenig gefährdet. Im Ausstreuungsbereich der Hustenden brauchen sie nur für kürzeste Fristen zu verweilen. Wohl atmen sie längere Zeit die Luft der mit Phthisikern belegten Krankensäle; aber nach den Ergebnissen aller darüber angestellten Untersuchungen wird es kaum jemals vorkommen, daB diese Luft so zahlreiche Tröpfchen (bzw. Stäubchen) mit Tuberkelbazillen enthält, daB die zur Infektion erforderliche Dosis zustande kommt. Die größeren mit Bazillen beladenen Tröpfchen setzen sich schon in etwa $1^{\mathrm{m}}$ Entfernung vom Hustenden zu Boden und die feinsten, vereinzelte Bazillen enthaltenden Schwebeteilchen werden auf sehr große Luftmengen rerteilt. - Vollends gelegentliche Unterhaltungen mit Phthisikern, Besuche in Sanatorien und Phthisikerkrankenhäusern, oder gemeinsames Essen, Spazierengehen und dgl. mit Phthisikern sind nicht geeignet, Gesunde mit Infektion zu bedrohen, falls nur die einfachsten Vorsichtsmaßregeln beobachtet werden. UnterläBt man freilich diese, so kann ausnahmsweise auch der Arzt und der gelegentliche Besucher eine zur Infektion ausreichende Bazillenmenge inhalieren. Die von mir beobachtete Frau Nic. z. B. verstreute an 3 verschiedenen Untersuchungstagen jedesmal so stark, daB recht wohl auch ein kürzerer Aufenthalt im Bereich ihrer Hustenstöße ausreichen konnte, um mehr als 400 Tuberkelbazillen in die Einatmungsluft überzuführen. Solche Fälle mögen bei den bekannt gewordenen Infektionen von Ärzten tatsächlich im Spiel gewesen sein. Da es dem Kranken schlechterdings nicht anzusehen ist, ob und in welchem MaBe er Tröpfchen verstreut, sollte in jedem Falle die Vorsicht, daB der Gesunde sich während des Hustens außerhalb des Bereichs der direkten Hustenstöße hält, beachtet werden. Erst auf Grund der quantitativen 
Feststellungen müssen wir eventuell unsere Vorstellungen von der Infektionsgefahr, die von der Tröpfcheninfektion ausgeht, modifizieren. Auf der einen Seite konnte sie bisher unterschätzt werden, weil man die Anzahl der in dieser Weise aufgenommenen Bazillen für belanglos ansah; viel hänfiger aber wurde die Tröpfcheninfektion überschätzt und dahin miBrerstanden, daB jedes kurze Zusammensein mit einem hustenden Phthisiker notwendig zu Infektionen führen mußte. Daraus entsprang ein. Mangel an Übereinstimmung mit den Erfahrungen der Praxis; man sah, $\mathrm{daB}$ sehr oft Gesunde mit Phthisikern verkehren, ohne angesteckt zu werden und man hielt dies für unmöglich, wenn die Tröpfcheninfektion zu Recht bestehen sollte. Und so wurden die Zweifel an der Bedeutung dieses Infektionsmodus um so stärker, je unbeschränlktere Wirksamkeit man ihm auf Grund der Experimente zuschrieb. Erst die Erkenntnis, daß offenbar eine gewisse Zahl von Tuberkelbazillen erforderlich ist, um Inhalationstuberkulose zu bewirken, und daß eine solche Zahl erst bei länger dauerndem Aufenthalt in nächster Nähe des hustenden Phthisikers durch Tröpfchenverstreuung in den Bereich der Einatmungsluft des Gesunden gelangt, läBt unsere praktischen Erfahrungen durchaus im Einklang mit den experimentellen Ergebnissen erscheinen.

Unter denjenigen Autoren, welche in letzter Zeit die Bedentung der Tröpfcheninfekton auf Grund der praktischen Erfahrungen völlig bestritten haben, ist namentlich Saugmann hervorzuheben, der durch eine sorgfältige Enquête das Verschontbleiben der Sanatorienärzte und der Kehlkopfärzte von Tuberkulose zu erweisen suchte and die Tröpfcheninfektion mit diesem Verschontbleiben für unvereinbar erklärte.

Saugmann (20) hat an eine große Zahl von an Lungenheilstätten beschäftigten Ärzten Fragebogen gesandt und zu ermitteln gesucht, ob diese Herren innerhalb ihrer Anstaltstätigkeit sich infiziert haben oder nicht. Er hat auf diese Weise von 180 Ärzten, die durchschnittlich 3 Jahre sich mit Tuberkulösen beschäftigt hatten und dann noch $3^{1} / 2$ Jahre beobachtet waren, verwertbare Antworten erhalten. Nicht selten flossen die Nachrichten über die Ärzte, über die berichtet wird, erst aus zweiter und dritter Quelle.

Von den 180 Ärzten, die geeignete Angaben machten, haben 9 angegeben, daß sie nach oder während ihrer Tätigkeit in Heilstätten tuberkulös erkrankt sind. Bei einem (Fall 5) handelt es sich anscheinend um eine Laboratoriumsinfektion mit Thimotheebazillen. Von den übrigen 8 Ärzten nimmt Saugmann an, sie hätten sich bis auf 2 schon vorher infiziert. Der Beweis für diese Meinung kann kaum als sicher erbracht angesehen werden und die Fälle lassen sich mit dem gleichen Rechte als Infektionen in den Anstalten auffassen. Es würde das also ein Zustande- 
krommen der Infeltion bei 4.4 Prozent der Ärzte bedeuten. - Mit einer solchen Zahl harmonieren auch andere Beobachtungen. Ich verweise hier nur auf die Statistik von Williams (37) über 180 Ärzte, die 1846 bis 1882 am Brompton Hospital für Lungenkranke tätig waren und von denen 9 tuberkulös wurden (also der gleiche Prozentsatz wie in der Saugmannschen Statistik). Ferner teilt Schaper (38) mit, daß in der Charité junge kräftige Ärzte, die sicherlich früher frei von Tuberkulose waren, des öfteren infiziert wurden. Ich selbst kenne ein Beispiel, das ich leider nicht des näheren ausführen darf, wo in einer allgemeinen Poliklinik im Laufe von 3 Jahren drei junge Årzte, die bei Antritt der Stellung völlig gesund waren, vermutlich infolge von Ansteckung tuberkulös wurden.

Es würde aber durchans unrichtig sein, wollte man gerade unter den Ärzten der Lungenheilstätten einen besonders hohen Prozentsatz von beruflich Infizierten erwarten. Wie ich oben bereits ausführte, werden die Ärzte durchaus nicht in besonders hohem Maße gefährdet, weil sie bei einiger Vorsicht nicht dauernd genug im Bereich stärkerer Tröpfchenverstreuung verweilen. Saugmann meint allerdings, $d a B$ dies bei der Untersuchung der Kranken doch der Fall sei, da ja auch seitlich von der Richtung der Hustenstöße und sogar hinter dem Patienten Tröpfchen mit Tuberkelbazillen von Heymann nachgewiesen seien. Saugmann übersieht dabei, daß es sich hier um vereinzelte, spärliche Tuberkelbazillen enthaltende Tröpfchen gehandelt hat, die für eine quantitativ ausreichende Infektion nicht in Betracht kommen. Im Gegenteil kommt der Arzt bei der ganzen Untersuchung des Patienten mit seiner Einatmung kaum für einen Moment in die gefährlichsten zentralen Teile des Ausstreuungsbereichs des Hustenden, vollends nicht während der Hustenstöße. Die Gefahr, in welcher der Arzt sich befindet, läßt sich jedenfalls gar nicht vergleichen mit derjenigen, in welcher sich z. B. das Pflegepersonal und Angehörige, vor allem Kinder, beim Zusammenleben mit dem Phthisiker befinden.

Ich gebe zu, daß Saugmann eine gröBere Gefährdung der Ärzte erwarten mußte, wenn er annahm, daß jedes verstreute Hustentröpfchen zur Infektion führen kann. Eine solche Ansicht ist eben nur mißverständlicherweise von einigen Autoren aus den Flüggeschen Versuchsergebnissen gefolgert. Flügge selbst hat stets darauf hingewiesen, daß zur richtigen Einschätzung der Infektionsgefahr eine genauere quantitative Kenntnis der Tröpfchenverstreuung gehöre. Berücksichtigt man aber die in dieser Richtung jetzt angestellten Untersuchungen, so entspricht die in der Praxis beobachtete Gefährdung der Sanatorienärzte durchaus den Vorstellungen, die wir aus den experimentellen Feststellungen über Umfang und Häufigkeit der Tröpfchenausstreuung uns bilden mußten. 
Saugmann hat aber noch ein anderes frappierenderes Ergebnis bei seiner Enquête erhalten. Er hat unter 64 Kehlkopfärzten keinen gefunden, der an Tuberkulose erkrankt ist. Schon Moritz Schmidt (39) hat in seinem Handbuch "Die Krankheiten der oberen Luftwege" das Verschontbleiben der Kehlkopfärzte hervorgehoben. Die Tatsache erscheint um so auffallender, als gerade für das Laryngoskopieren die Verstreuung Tuberkelbazillenhaltiger Tröpfchen durch mehrfache Untersuchungen festgestellt ist. Sa u g mann selbst teilt mit, daß er z.B. an seinen Brillengläsern nach dem Laryngoskopieren Tuberkelbazillen hat nachveisen können; ähnliche Beobachtungen machte B. Fränkel (22). Die genauesten Untersuchungen rühren von Möller (23) her. DaB dieser am Kehlkopfspiegel Sputumteilchen mit Tuberkelbazillen fand, ist ohne Belang; aber auch an dem Reflektor, an der Lehne seines Stuhls usw. fand er nach dem Laryngoskopieren Tuberkelbazillenhaltige Tröpfchen. Ferner konstatierte er bei 75 Untersuchungen des eigenen Nasenschleims (nach $2 \frac{1}{2}$ stündiger Sprechstunde mit zahlreichen Lungen- und Kehlkopfuntersuchungen) einmal einen T.B., einmal 4 , und ein drittes Mal ein Häufchen von 6 bis 8 Bazillen.

Zweifellos findet also beim Laryngoskopieren Tröpfchenverstreuung seitens des Kranken statt. — Aber auch hier wird erst eine Berüeksichtigung der quantitativen Verbältnisse zu einem richtigen Urteil führen, und um in dieser Richtung einen genaueren Einblick zu gewinnen, stellte ich selbst noch eine Reihe von Untersuchungen an.

Ich ging zu diesem Behufe in der Weise vor, daB ich in ganz ähnlicher Weise wie bei den schon besprochenen Verstreuungsversuchen, 12 Objektträger, die zusammen einen Raum von $324 \mathrm{qcm}$ einnahmen, in einen Rahmen einspannte, der durch Bänder leicht in frontaler Stellung an dem Reflektor des Untersuchers angebracht werden konnte. Bei einiger Übung konnte man durch die Gläser hindurch ganz gut laryngoskopieren und intralaryngeale Eingriffe vornehmen. Das Gesicht des Untersuchers war so völlig geschützt und die beim Husten etwa versprayten Tröpfchen wurden auf den Objektträgern aufgefangen.

Nach jedem Spiegelversuche wurden die Objektträger vorsichtig fixiert und weiterhin, wie schon im ersten Teile der Arbeit beschrieben, behandelt.

Die Versuche beim Kehlkopfspiegel konnte ich dank der Güte des Herrn Professor Dr. Hinsberg zum großen Teile in der hiesigen Universitätspoliklinik für Kehlkopfkranke anstellen. Ihm, sowie seinen Assistenten bin ich für ihre freundliche Hilfe sehr zu Dank verpflichtet. Einer der Assistenten, Herr Dr. Freytag, ist leider inzwischen an einer Phthise gestorben, die schon vor seiner laryngologischen Tätigkeit bestanden hat. 
Da die Versuche alle in übereinstimmender Weise ausfielen, möchte ich hier nur zur Erläuterung meines Vorgehens ein Versuchsprotokoll ausführlich wiedergeben, im übrigen aber meine Resultate in einer Tabelle zusammenfassen.

\section{Versuch IV.}

\section{Untersucher Dr. S. Patient: Frau S. Z.}

24.III. 05. Ausgedehnter Lungenbefund. Die gesamte Larynxwand verdickt. Ulcerationen auf der hinteren Larynxwand. Beide Stimmbänder therapeutisch verschorft.

Therapeutischer Eingriff: Kokainisieren, Ätzung der Uloerationen mit Milchsäure.

\begin{tabular}{|c|c|c|}
\hline $5 \cdot 59$ & $\underline{0}^{\prime \prime}$ & \\
\hline $6 \cdot \overline{0^{\prime}}$ & $10^{\prime \prime}$ & , \\
\hline $6 \cdot \overline{0^{\prime}}$ & $\overline{30}^{\prime \prime}$ & $n$ \\
\hline $6 \cdot \overline{0^{\prime}}$ & $\overline{40}^{\prime \prime}$ & " \\
\hline $6 \cdot 1^{\prime}$ & $\overline{10}^{\prime \prime}$ & " \\
\hline $6 \cdot 1^{\prime}$ & $\overline{15}$ & $"$ \\
\hline $6 \cdot 1^{\prime}$ & $\overline{40}^{\prime \prime}$ & $"$ \\
\hline $6 \cdot \mathbf{2}^{\prime}$ & $\overline{\mathbf{4 5}}^{\prime \prime}$ & $"$ \\
\hline $6 \cdot \mathbf{2}^{\prime}$ & $\underline{54} \underline{5}^{\prime \prime}$ & \\
\hline
\end{tabular}

\begin{tabular}{|c|c|c|c|}
\hline Zweimal & lautiert. & Dauer & $5 \mathrm{Sek}$ \\
\hline Hustet & stark. & $n$ & $2 \eta$ \\
\hline$"$ & $"$ & $"$ & 2 \\
\hline Hustet & $n$ & $"$ & \\
\hline Zweimal & lautiert & $n$ & 5 \\
\hline $\begin{array}{l}\text { Gehustet } \\
\text { Einmal I }\end{array}$ & & $"$ & 5 \\
\hline $\begin{array}{l}\text { Einmal } \\
\text { Einmal }\end{array}$ & $\begin{array}{l}\text { lautiert } \\
\text { gehustet }\end{array}$ & $n$ & \\
\hline $\begin{array}{l}\text { Laut } \\
\text { Starker }\end{array}$ & $\begin{array}{l}\text { tiert. } \\
\text { Husten }\end{array}$ & & \\
\hline
\end{tabular}

Die Durchschnittsentfernung des Arztes vom Kranken beträgt etwa $40 \mathrm{~cm}$.

Die Gesamtdauer des Eingriffes beträgt 3 Min. 54 Sek. Die Dauer tatsächlichen intralaryngealen Arbeitens 31 Sekunden.

Die Zusammenstellung der auf dem Wege ähnlicher Versuche gewonnenen Befunde gibt Tabelle II.

Es ist überraschend zu sehen, wie relativ wenig Ausbeute an Tröpfchen und Bazillen die Spiegelversuche gegeben haben.

Besonders deutlich tritt das bei Versuchen 63 bis 67 zutage, die an Personen vorgenommen wurden, die ich schon vorher auf ihr Verstreuungsvermögen (vgl. Tabelle I, Nr. 49 bis 51,59 bis 61 und 27 bis 42 ). untersucht hatte. Während alle drei Versuchspersonen bei kräftigem Husten Bazillen verstreut hatten, zum Teil sogar außerordentlich viel (Fall Nic.) konnte bei den Spiegelversuchen nur einmal e in Bacillus nachgewiesen werden. Auch die Zahl der verstreuten Tröpfchen war sehr klein, Bronchialtröpfchen fehlten fast ganz.

Aber auch bei den. Kehlkopfphthisikern, die ich zu untersuchen Gelegenheit hatte, war ein gleiches Verhalten $\mathrm{zu}$ bemerken. Nur einige Male konnte ich vereinzelte Tuberkelbazillen nachweisen, während im Sputum bei allen Patienten mehr oder minder zahlreiche Bazillen gefunden waren. 
Dieses zunächst überraschende Ergebnis findet seine Erklärung im Verhalten des Untersuchers sowohl, als auch auch in besonderen Verhältnissen des Kranken.

Was den Untersucher anlangt, so wendeten alle Kehlkopfärzte, die ich darauf hier $\mathrm{zu}$ beobachten Gelegenheit hatte, fast unbewuBt gewisse Vorsichtsmaßregeln an.

Im Augenblick, wo der Arzt merkt, daB der Untersuchte husten will, weicht er zurück. Der Gespiegelte, der dem unangenehmen Reiz zu entgehen trachtet, nimmt seinen Kopf auch zurück; so wird im Momente des Hustens der Abstand zwischen Arzt und Patient bis auf mindestens 80 bis $90^{\mathrm{em}}$ vergrößert. Außerdem wendet der Arzt seinen Kopf im entscheidenden Moment zur Seite und bringt ihn so zum gröBten Teile auBerhalb des Zerstreuungskreises des Hustenden. Der untersuchende Arzt hat sehr bequem die Zeit, sich im rechten Augenblicke sozusagen in Sicherheit zu bringen, denn er sieht die gefahrdrohende Larynxkontraktion im Spiegel. Ferner habe ich bei fast allen Kehlkopfärzten bemerkt, daB sie im entscheidenden Momente den Atem instinktiv, häufig ohne sich dessen selbst bewußt zu werden, anhielten und auch dadurch die Inhalationsgefahr verminderten.

Alle diese, in fast jedem Falle zu beobachtenden MaBnahmen können aber den geringen Bazillenbefund nicht erklären. Es müssen noch andere Faktoren mit im Spiele sein, und diese liegen in der Person des während des Kehlkopfspiegelns hustenden Phthisikers selbst.

Um die hier in Frage kommenden Verhältnisse klarlegen zu bönnen, muB ich kurz auf die Mechanik der Expektoration und Physiologie des Hustens eingehen.

Die Expektoration ist die Folge von drei verschiedenen Vorgängen, der Tätigkeit der Flimmerepithelien des Respirationstraktes, der Wirksamkeit der sich peristaltisch kontrahierenden Bronchialmuskulatur [Henle (40)] und endlich jenes reflektorisch ablaufenden Bewegungskomplexes, den wir als Husten bezeichnen.

Sind die Sekretmassen erst in die feineren Bronchi gelangt, so werden sie in stiller, andauernder Arbeit von den Flimmerepithelien oralwärts geschafft, die in ihrer Wirkung von der Peristaltik der Bronchialmuskulatur unterstützt werden. Durch einen Reiz im Bronchus und der Trachea wird nun die gewaltsame, durch Glottisschlub zunächst behinderte Exspiration herbeigeführt, die wir Husten nennen. Durch den zeitweisen Glottisverschluß wird der intratracheale Druck, der für die Herausbeförderung der Sekretmassen von entscheidender Bedeutung ist, wesentlich erhöht. Nun pfeift im Nomente, wo der GlottisverschluB gelöst und die Bahn wieder frei wird, die Luft unter kräftigem Druck heraus und reißt 
die Sekretmassen mit sich fort. Das ist der Vorgang beim typischen Husten.

Der Husten kann aber auch ohne GlottisverschluB zustande kommen, wie Beobachtung am tracheotomierten Tiere und Menschen, sowie die beim Laryngoskopieren gewonnene Erfahrung zeigt. Bei fehlendem oder 'mangelhaftem GlottisverschluB ist der Fxspirationsdruck in der Trachea, der für die Herausbeförderung der Selkretmassen vor allem wichtige Intratrachealdruck weit geringer als in der Norm. Aron (41) hat Gelegenheit gehabt, hierüber beim tracheotomierten Menschen, bei dem dieselben Verhältnisse vorliegen wie beim fehlenden GlottisschluB Messungen anzustellen. Er fand den Druck bei einem und demselben Menschen verschieden, je nachdem dieser durch den Larynx oder durch die Trachealfistel atmet.

\begin{tabular}{c|c}
\hline Atmung durch Larynx & Atmung durch Trachealfistel \\
\hline$+78.8 \mathrm{~mm} \mathrm{Hg}$ & $+23 \cdot 0 \mathrm{~mm} \mathrm{Hg}$ \\
76.6 & $\mathbf{3 4 . 8}$ \\
94.2 & 51.6 \\
91.2 & 42.0 \\
80.4 & 40.6 \\
89.2 & 42.8 \\
85.0 & 50.4 \\
82.0 & 40.6 \\
& 48.0
\end{tabular}

Die Unterschiede liegen klar zutage; beim Atmen durch den Larynx beträgt der Maximalwert 94.2, beim Atmen durch die Trachealwunde $51.6 \mathrm{~mm} \mathrm{Hg}$. Tatsächlich dürfte der intratracheale Druck des gesunden Menschen aber noch höher sein, wie aus Beobachtungen von Geigel (42) hervorgeht, der Werte von 150 bis $160 \mathrm{~mm} H g$ feststellen konnte. Der intratracheale Druck beim gesunden mit GlottisschluB atmenden Menschen kann also unter Umständen bis achtmal gröBer sein als beim kranken, der mit fehlendem oder mangelhaftem GlottisschluB hustet; es wird somit das Husten unter letzteren Umständen auch einen achtmal geringeren Expektorationswert haben als bei normalen Verbältnissen.

Betrachten wir nun das Husten der Kehlkopfphthisiker. Wie husten sie, wie verhält sich ihr Larynxverschluß? Für einen großen Teil der Kranken - solche mit Ulcerationen an den Taschen- und Stimmbändern oder mit Infiltraten an der hinteren Larynxwand ist ein normaler GlottisrerschluB ein Ding der Unmöglichkeit.

Aber auch allen anderen Patienten, die wir kehlkopfspiegeln, erschweren wir den festen GlottisversehluB sehr durch unsere VerhaltungsmaBregeln. Wir pflegen ihnen zu sagen: „Atmen Sie ruhig und tief, damit Sie nicht 
zu husten und nicht zu würgen brauchen." Nun sitzen die Leute mit weitgeöffneter Glottis da, und sobald wir merken, daB eine Würgbewegung entsteht, halten wir sie wieder zum ruhigen Atmen an. Die Kranken vermeiden aber auch schon von selbst den GlottisschluB nach Kräften, weil er der Anfangspunkt der reflektorischen Würgbewegung ist, die ihnen natürlich unangenehm ist. Kommt es zum festen Glottisverschlub, so folgt fast sicher eine heftige Würgbewegung, infolge welcher der Patient zurückweicht und die Untersuchung unterbrochen werden muB.

Es kommt somit beim Patienten, der laryngoskopiert wird, nur relativ selten zu einem plötzlichen unvorhergesehenen Husten; meist erfolgt dieser dann mit mangelhaftem oder fehlendem Glottisschlub. Kommt es aber auch einmal zu einem richtigen, kräftigen Hustenstoße, so sieht der spiegelnde Arzt dies schon vorher im Kehlkopfspiegel und hat, da er ja sowieso wegen der darauf folgenden Würgbewegungen die Untersuchung oder den therapeutischen Eingriff unterbrechen muB, Zeit genug, sich zur Seite zu wenden und aus dem Bereiche des gefährdenden Verstreuungskegels zu bringen.

Hustet der Kranke mit mangelndem GlottisschluB, so ist die notwendige Folge davon, daB er anch viel weniger heraushustet und viel weniger Bazillen verstreut.

Letztere können stammen: 1. aus den Bronchien, 2. vom schleimigeitrigen Belege der Larynxulcerationen und 3. aus dem Munde. Die Exspirationskraft ist aber bei dem mangelhaften Husten im allgemeinen $\mathrm{zu}$ gering, um Bronchialtröpfchen heraufzubringen, mindestens sind sie außerordentlich selten. Der Beleg der Kehlkopfulcera ist von äuBerst zäher, eitrig-schleimiger Beschaffenheit. Wir wissen aus der Arbeit Stichers (15), welch große Kraft notwendig ist, aus solchem Schleim Tröpfchen frei zu machen. Somit bleiben vorzugsweise nur noch die Mundtröpfchen, die in gewisser Zahl verstreut werden, die aber, wie wir schon im ersten Teil unserer Untersuchungen gesehen haben, sehr selten Tuberkelbazillen enthalten. Die geringe Ausbeute, die wir in unseren Spiegelversuchen hatten - im ganzen nur drei Tuberkelbazillen - entspricht durchaus diesen Anschauungen. Tatsächlich ist also die Infektionsgefahr für die Kehlkopfärzte bei weitem nicht so groB, als man es von vornherein anzunehmen geneigt ist. - Natürlich kann auch ein Kehlkopfarzt sich infizieren; bleibt er nach der Beendigung der therapeutischen Eingriffe, wo der Patient meist einigemale kräftig zu husten pflegt, noch eine Zeitlang in nächster Nähe des Kranken und im Bereich von dessen HustenstöBen, so wird er zweifellos das nötige Quantum Bazillen einatmen können. Aber wenn eine solche unnötige Annäherung vermieden und die einfachsten VorsichtsmaBregeln beobachtet werden, erreicht die Zahl der in verstreuten Tröpfchen enthaltenen Bazillen nicht die infektiöse Dosis. Das zeigen nicht 
nur meine Versuche, sondern ebensowohl die von Moeller angestellten, der ja z. B. im eigenen, nach der Sprechstunde sorgfältig untersuchten Nasenschleim 72 mal keinen einzigen Bazillus, und 3 mal nur vereinzelte fand.

Im ganzen sollte man die Beweiskraft einer solchen Enquête, wie sie Saugmann veranstaltet hat, nicht zu hoch veranschlagen. Sa ugmann versandte Fragebogen an fünfzig laryngologische Polikliniken; nur aus acht bekam er die erbetenen vollständigen Ärztelisten. Es liegt doch sehr nahe daran zu denken, dab ein Teil der Polikliniken, die nicht geantwortet haben, unangenehme Erlebnisse oder wenigstens zweifelhafte Resultat ein bezug auf die Infeition ihrer Ärzte zu verzeichnen hatten und deshalb nicht antworteten. Kommt eine berufsmäBige Infektion zustande, so kann der Verdacht entstehen, daB die erforderlichen VorsichtsmaBregeln in der betreffenden Poliklinik nicht richtig gehandhabt sind; und einer solchen Kritik wird sich der Leiter des Instituts selbst dann nicht gern aussetzen, wenn der Fall zweifelhaft liegt und nach seiner Utherzeugung die Erkrankung schon vor der laryngoskopischen Tätigkeit entstanden ist. In dem ron mir erwähnten Fall, wo ein Assistent der Breslauer Poliklinik an Phthise starb, bin ich nicht zu Diskretion verpflichtet worden, weil die frühere Infektion sicher war. Von den anderen oben ron mir zitierten Erkrankungen von Assistenten, wo die berufliche Infektion keineswegs auszuschließen war, habe ich nur unter der Zusicherung völliger Verschwiegenheit genauere Nachricht erhalten. Es ist daher nicht von der Hand zu weisen, daB das von Sa ug man $n$ gesammelte Material a u sgewählt und deshalb unbrauchbar ist; warum sollte denn unter einer so großen Zahl von Ärzten, wie sie sich ergibt, wenn man alle Gefragten, auch diejenigen, welche nicht geantwortet haben, mitzählt, während der Jahre ihrer Berufstätigkeit nicht Einer, wenn auch durch Infektion außerhalb des Berufs, an Tuberkulose erkranken?

Mit derartigen Einwänden kann die Bedeutung der Tröpfcheninfeltion bei der Phthiseverbreitung nicht erschüttert werden. Die Leichtigkeit und Sicherheit, mit der Meerschweinchen und andere Versuchstiere durch versprengte Tröpfchen mit Tuberkelbazillen infiziert werden können; die Tatsache, daB die Phthisiker reichlich feinste Tröpfchen mit Tuberkelbazillen verstreuen; die mehrfach gelungenen Versuche, Meerschweinchen durch direktes Anhusten von Phthisikern zu infizieren; endlich die analoge Rolle der infektiōsen Tröpfchen bei Lungenpest, Influenza und anderen infektiösen Katarrhen, Diphtherie, Genickstarre usw., wo eine Verbreitung durch trockene Stäubchen wegen der Widerstandslosigkeit der Erreger ganz ausgeschlossen ist - alles dies gibt zusammen ein so gewichtiges Beweismaterial, daB nur eine fanatische Gegnerschaft die Bedeutung der Tröpfcheninfektion leugnen kann. 
H. ZIESCHE:

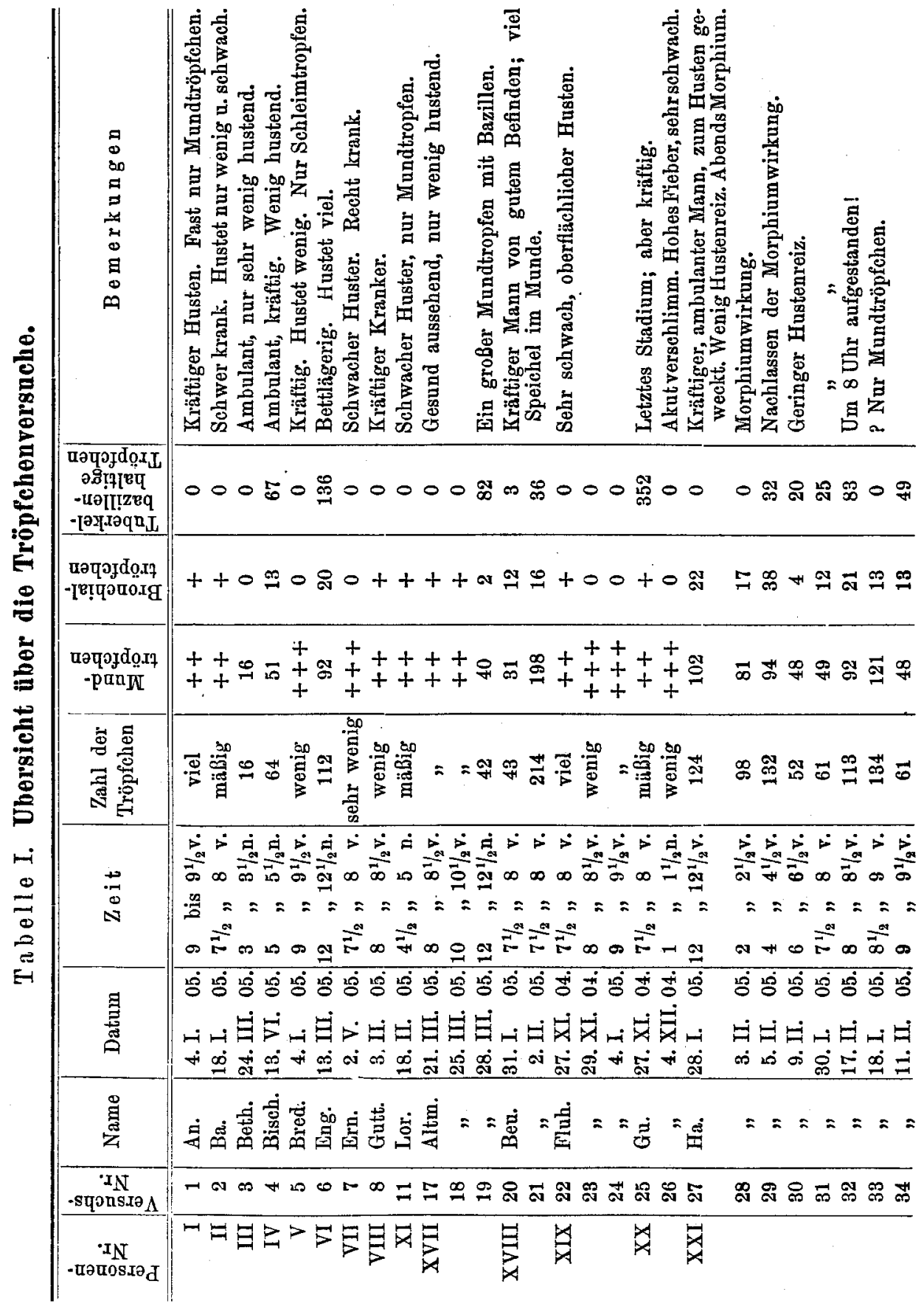


Über Tröpfchenadsstredung DURCh hustende Phthistker. 77

\begin{tabular}{|c|c|}
\hline 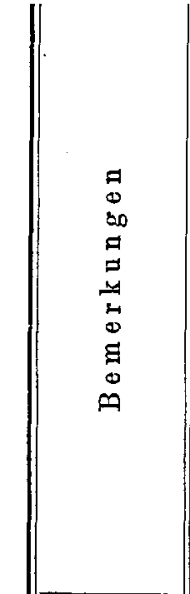 & 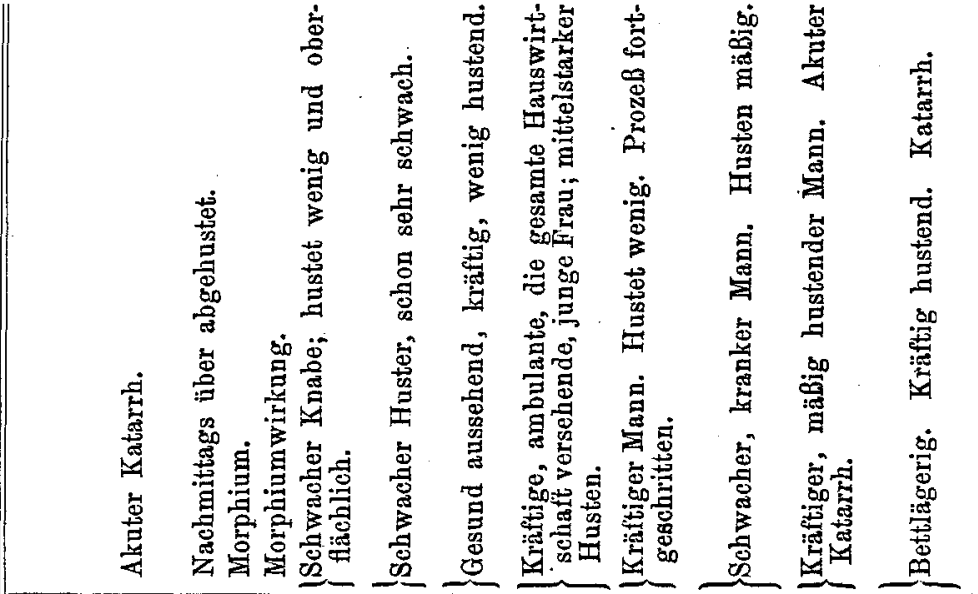 \\
\hline 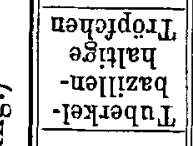 & 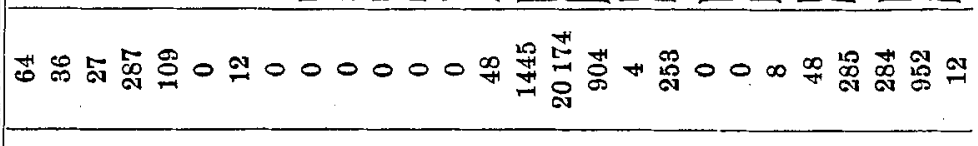 \\
\hline 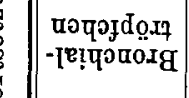 & 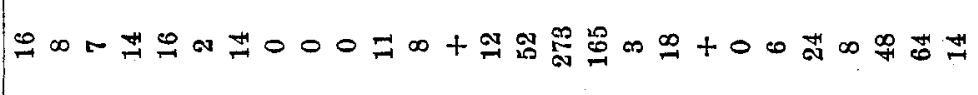 \\
\hline 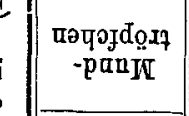 & 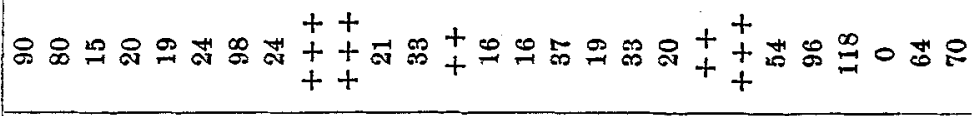 \\
\hline 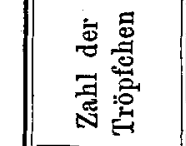 & 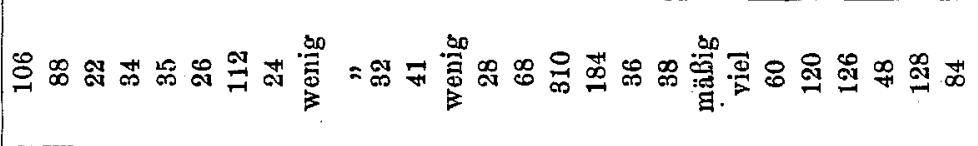 \\
\hline 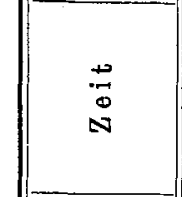 & 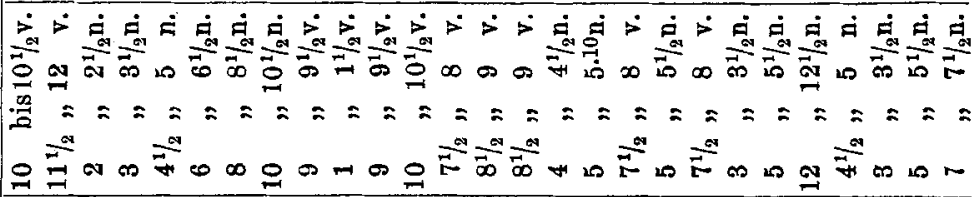 \\
\hline 嗖 & 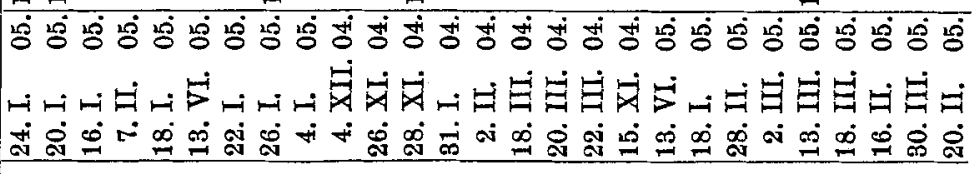 \\
\hline 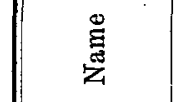 & 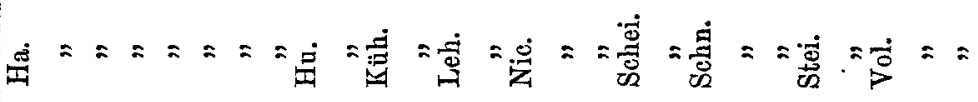 \\
\hline $\begin{array}{c}\text { in } \\
-\operatorname{sqonsia} \\
\end{array}$ & 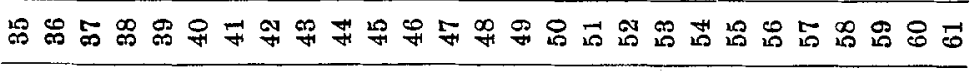 \\
\hline 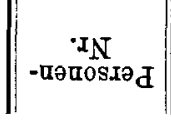 & 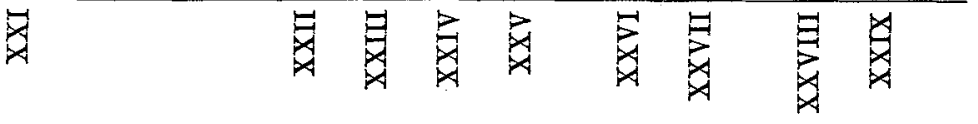 \\
\hline
\end{tabular}




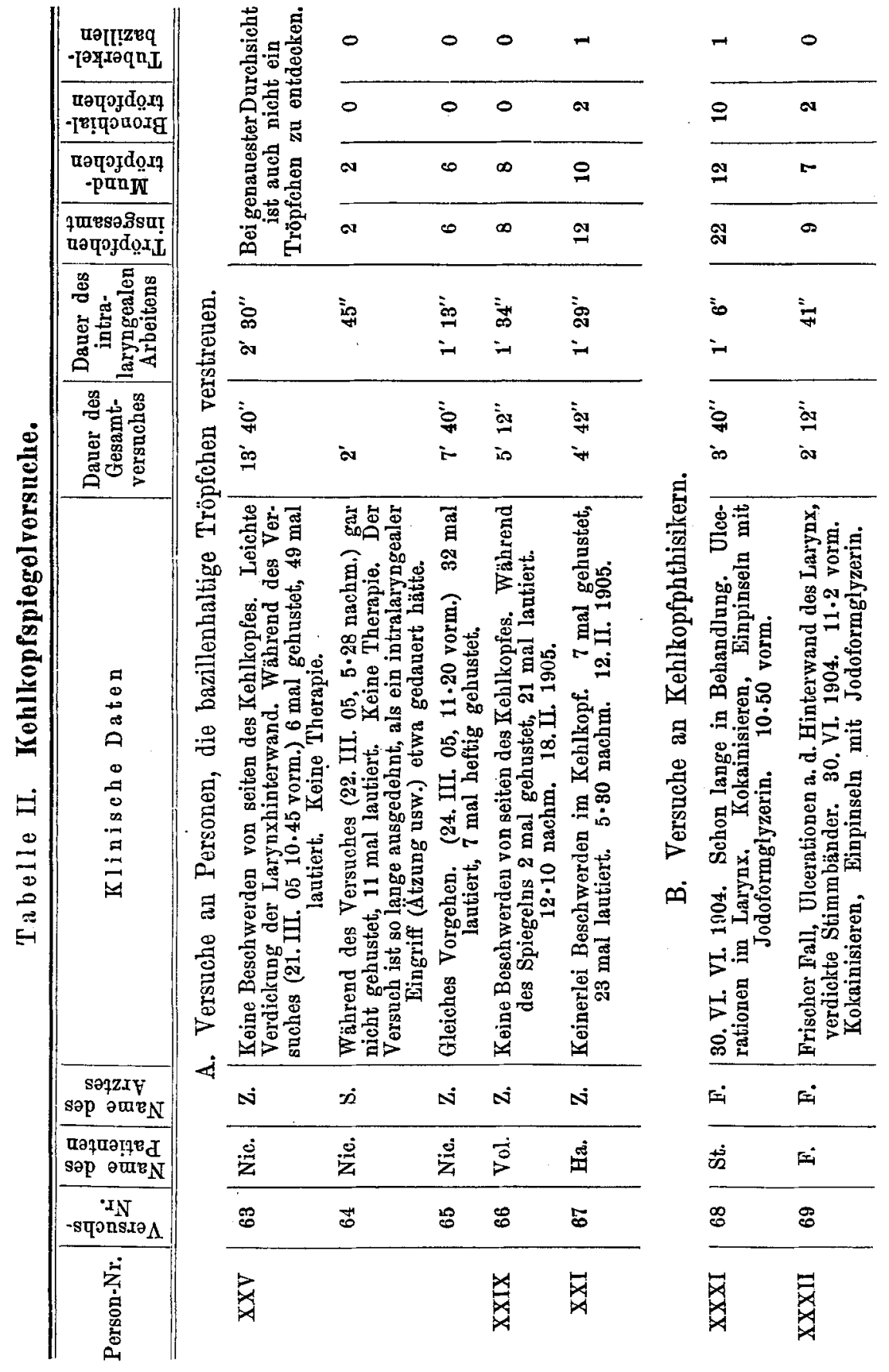


Über TröPfchenausstredung dURCh hustende Phthisiker.

\begin{tabular}{|c|c|c|c|c|c|c|c|}
\hline 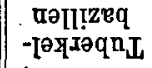 & $\pi$ & 0 & 0 & 0 & 0 & 0 & 0 \\
\hline 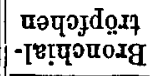 & $\infty$ & ㅇ & 0 & 0 & 0 & 0 & N \\
\hline 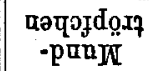 & $\infty$ & 0 & a & D. & $\infty$ & + & $\infty$ \\
\hline 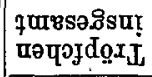 & $\infty$ & $\sigma$ & $\infty$. & nos & (מ & $H$ & 10 \\
\hline 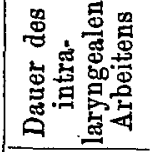 & $\begin{array}{l}\text { is } \\
\text { in }\end{array}$ & $\begin{array}{l} \pm \\
=\end{array}$ & $\begin{array}{l}\text { 휴 } \\
\text { = }\end{array}$ & : & 엄 & is & 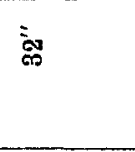 \\
\hline 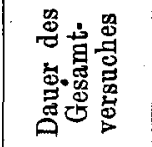 & in & 三 & is & is & $\begin{array}{l}\text { 함 } \\
\text { in }\end{array}$ & $\begin{array}{l}\text { 음 } \\
\text { - }\end{array}$ & $\begin{array}{l}\text { is } \\
\text { i- }\end{array}$ \\
\hline 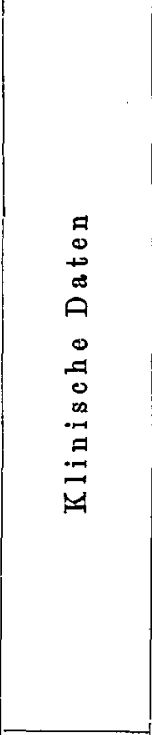 & 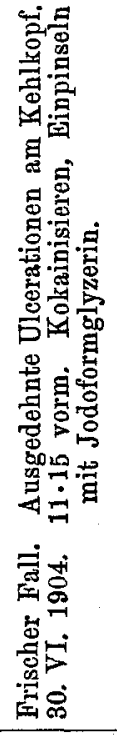 & 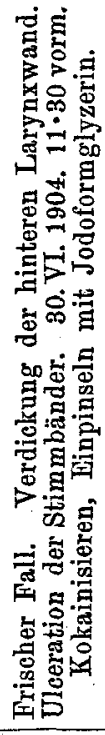 & 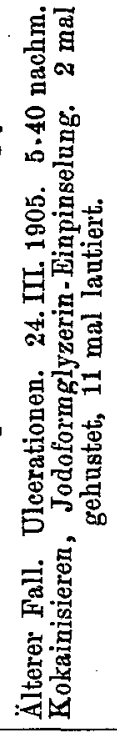 & 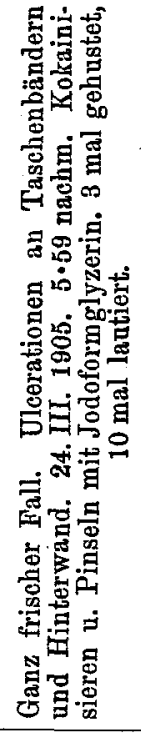 & 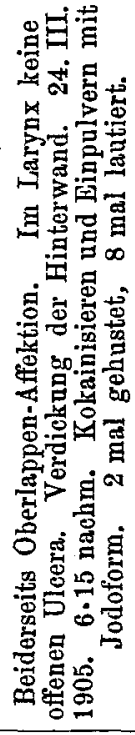 & 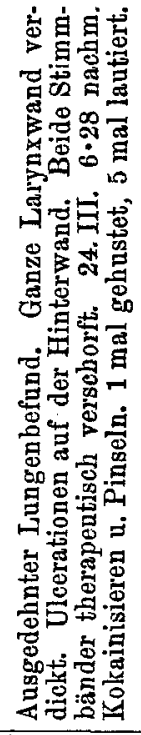 & 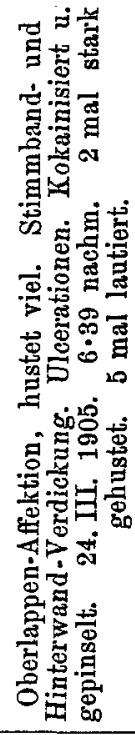 \\
\hline $\begin{array}{c}\text { seqzIV } \\
\text { səp әшा'N }\end{array}$ & $F^{\circ}$ & 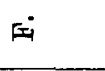 & $\dot{N}$ & $\dot{\theta}$ & $\nabla \dot{0}$ & $\ddot{\theta}$ & $\dot{x}$ \\
\hline 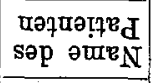 & ن & $\dot{D Q}$ & 乎 & v & مُ & $\approx$ & $\approx$ \\
\hline 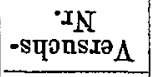 & 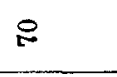 & $\vec{F}$ & $\stackrel{\mathbf{N}}{\mathbf{S}}$ & $\stackrel{\infty}{0}$ & $\underset{F}{*}$ & $\stackrel{20}{2}$ & 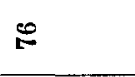 \\
\hline $\begin{array}{l}\dot{\dot{z}} \\
\dot{\dot{0}} \\
\dot{0} \\
\dot{0} \\
\end{array}$ & 园 & 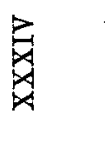 & $\overrightarrow{\not x}$ & 必 & 是 & 罗 & $\begin{array}{l}\not{x} \\
x \\
x\end{array}$ \\
\hline
\end{tabular}


Als ein Versuch, zum besseren Verständ̉nis der Tröpfcheninfeiztion beizutragen, ist auch die vorliegende Arbeit anzusehen, deren Hauptinhalt sich folgendermaBen zusammenfassen läßt:

1. Bei einmaliger Untersuchung finden sich unter Phthisikern, deren Sputum Tuberkelbazillen enthält, nur 30 bis 40 Prozent, die beim Husten Tröpfchen verstreuen. Bei wiederholten Untersuchungen derselben Patienten steigert sich dieser Prozentsatz erheblich.

2. Die Tröpfchen entstammen teils der Mundflüssigkeit und enthalten dann selten Tuberkalbazillen; teils liegen Bronchialtröpfchen vor, die sehr häufig tuberkelbazillenhaltig und oft sehr reich daran sind.

3. Die binnen einer halben Stunde auf einer in 40 bis $80 \mathrm{~cm}$ Entfernung aufgestellten Glasplatte aufgefangenen Tröpfchen enthalten in etwa 20 Prozent der Untersuchungen über 400 und bis 20000 Tuberkelbazillen; in 80 Prozent keine oder weniger als 400 Bazillen.

4. Auf Grund der von Gebhard, Preyss, Findel an Versuchstieren angestellten Experimente werden wir annehmen müssen, daB mindestens 200 bis 400 Tuberkelbazillen, vielleicht sogar noch mehr, in der Einatmungsluft erforderlich sind, um beim Menschen eine Infektion herrorzurufen.

5. Demnach erfolgt eine Infektion durch Tröpfchenverstreuung nicht bei kurzdauerndem Zusammensein mit einem Phthisiker; nicht wenn der Gesunde den Bereich der direkten HustenstöBe und ein Nahekommen auf mehr wie einen Meter vermeidet, oder wenn der Hustende sich während der HustenstöBe abwendet. Ärzte sind auch bei der Untersuchung ron Phthisikern nur wenig gefährdet.

6. Dagegen führt das dauernde enge Zusammensein von Mutter und Kind häufig zur Infektion; weniger häufig ist dies bei Eheleuten der Fall. Krankenpfleger sind namentlich bei bettlägerigen, hilflosen Phthisikern der Tröpfcheninfektion stark exponiert.

7. Kehlkopfärzte sind bei den anscheínend gefährdenden Untersuchungen und therapeutischen Eingriffen relativ wenig durch Tröpfeheninfektion gefährdet, weil der Kranke bei offener Glottis wenig Tuberkelbazillen verstreut und weil der Arzt im übrigen in der Lage und gewöhnt ist, sich der Aufnahme ausgehusteter Bronchialtröpfchen zu entziehen. 
Über Tröpfchenausstreudng DURCh hustende Phthisiker. 81

\section{Literatur-Verzeichnis.}

1. Tappeiner, Virchows Archiv. 1878. Bd. LXXIV. S. 393. - Ebenda. 1880. Bd. LXXXII. S. 353. - Archiv für klin. Medizin. 1884. Bd. XXIX. S. 595.

2. Franz, Wiener klin. Wochenschrift. 1904. Nr. 16. S. 1028.

3. Naegeli, Virchows Archiv. 1900. Bd. CLX. S. 426.

4. Villemin, Étude expér. et clin. sur la tub. Paris 1868.

5. v. Eiselsberg, Wiener med. Wochenschrift. 1887. S. 1729.

6. Holst, Lancet. 1886. II. p. 743.

7. Merklen, Rev. des scienc. médic. 1888. Nr. 52.

8. Tscherning, Fortschritte der Medizin. 1885. S. 65.

9. Volland, Zeitschrift für klin. Medizin. Bd. XXIII.

10. Baldwin, Transact. of Americ. climatolog. associat. 1898.

11. Dieudonné, Münchener med. Wochenschrift. 1901. Nr.48. S. 1439,

12. Cornet, Diese Zeitschrift. 1889. Bd.V. S. 192. - 1889. Bd. VI. S. 65. 1891. Bd. X. S. 455 .

13. Flügge, Deutsche med. Wochenschrift. 1897. Nr. 23. S. 665, 758. - Diese Zeitschrift. 1897. Bd. XXV. S. 179. - 1899. Bd. XXXIII. S. 107. - 1901. Bd. XXXVIII. S. 1 .

14. Neisser, Diese Zeitschrift. 1898. Bd. XXVII. S. 175.

15. Sticher, Ebenda. 1899. Bd. XXX. S. 163.

16. Petterson, Nord. medic. Ark. 1901. Abt. II. S. 163.

17. Gotschlich, Inaug.-Dissertation. Breslau 1902.

18. Laschtschenko, Diese Zeitsehrift. 1899. Bd. XXX. S. 125.

19. Heymann, Ebenda. 1899. Bd. XXX. S. 139. - 1901. Bd. XXXVIII. S. 21.

20. Saugmann, Zeitschr. f. Tuberk. u. Heilst. Bd. VI. S. 125.

21. Engelman n, Inaug.-Dissertation. Berlin 1898.

22. Fraenkel, Berline" klin. Wochenschrift. 1899. S. 21.

23. Moeller, Diese Zeitschrift. 1899. Bd. XXXII. S. 203.

24. Koeniger, Ebenda. 1900. Bd. XXXIV. S. 119.

25. Bl a me, Berliner klin. Wochenschrift. 1905. Nr. 42. S. 1072.

26. Gebhard, Virchows Archiv. 1890. Bd. CXIX. S. 127.

27. Preyss, Münchener med. Wochenschrift. 1891. S. 418.

28. Barthel, Wiener klin. Wochenschrift. 1906. S. 217.

29. Mosny, Ann. d'hygièn. publ. 1902. T. XLVII. p. 289.

30. Mosny, Ann. d'hygièn. publ. 1904. T. IV. p. 15.

Zeitschr. f. Hggiene. LVIL. 
31. Sorgo, Wiener klin. Wochenschrift. 1904. Nr. 17. S. 725.

32. Brouardel et Gilbert, Nouveau traité de médecine et de thérapeutique publié en fascicules. Paris, Baillière et fils, 1906. - Bernard et Mosny, Tuberculose. 1906. Fasc. 4 . p. 97 u. 100.

33. Hillier, Brit. med. Joum. 1903. p. 592.

34. Kirstein, Diese Zeitschrift. 1900. Bd. XXXV. S. 123. - 1905. Bd. L. S. 186.

35. Boeg, Ebenda. 1904.

36. L. H. Bing, Norsk magazin for Lagevidenskaber. 1904. Nr. 82.

37. Willia ms, Brit. med. Journ. Sept. 1882.

38. Schaper, Tuberculosis. 1904. Bd. II. p. 543.

39. M. Schmidt, Lehrbuch der Laryngologie.

40. Henle, Zeitschrift für ration. Medizin. 1844. Bd. I. S. 249.

41. Aron, Virehows Archiv. 1892. Bd. CXXIX. S. 429. - Zeitschr, f. klin. Medizin. 1904. Bd. LIV. S. 136.

42. Geigel, Virchows Archiv. 1900. Bd. CLXI. S. 183.

43. v. Behring, Kasseler Vortrag, Marburg 1903. - Deutsche med. Wochenschr. 1903. S. 689.

44. Petterson, Nord. med. Ark. 1900. Bd. XXXIII p. 1. 